\title{
Iterative Schemes for Convex Minimization Problems with Constraints
}

\author{
Lu-Chuan Ceng, ${ }^{1}$ Cheng-Wen Liao, ${ }^{2}$ Chin-Tzong Pang, ${ }^{3}$ and Ching-Feng Wen ${ }^{4}$ \\ ${ }^{1}$ Department of Mathematics, Shanghai Normal University, and Scientific Computing Key Laboratory of Shanghai Universities, \\ Shanghai 200234, China \\ ${ }^{2}$ Department of Food and Beverage Management, Vanung University, Chung-Li 320061, Taiwan \\ ${ }^{3}$ Department of Information Management and Innovation Center for Big Data and Digital Convergence, Yuan Ze University, \\ Chung-Li 32003, Taiwan \\ ${ }^{4}$ Center for Fundamental Science, Kaohsiung Medical University, Kaohsiung 807, Taiwan
}

Correspondence should be addressed to Chin-Tzong Pang; imctpang@saturn.yzu.edu.tw

Received 17 February 2014; Accepted 12 April 2014; Published 8 May 2014

Academic Editor: Xie-ping Ding

Copyright (C) 2014 Lu-Chuan Ceng et al. This is an open access article distributed under the Creative Commons Attribution License, which permits unrestricted use, distribution, and reproduction in any medium, provided the original work is properly cited.

We first introduce and analyze one implicit iterative algorithm for finding a solution of the minimization problem for a convex and continuously Fréchet differentiable functional, with constraints of several problems: the generalized mixed equilibrium problem, the system of generalized equilibrium problems, and finitely many variational inclusions in a real Hilbert space. We prove strong convergence theorem for the iterative algorithm under suitable conditions. On the other hand, we also propose another implicit iterative algorithm for finding a fixed point of infinitely many nonexpansive mappings with the same constraints, and derive its strong convergence under mild assumptions.

\section{Introduction}

Let $C$ be a nonempty closed convex subset of a real Hilbert space $H$ and let $P_{C}$ be the metric projection of $H$ onto $C$. Let $S: C \rightarrow H$ be a nonlinear mapping on $C$. We denote by $\operatorname{Fix}(S)$ the set of fixed points of $S$ and by $\mathbf{R}$ the set of all real numbers. A mapping $V$ is called strongly positive on $H$ if there exists a constant $\bar{\gamma}>0$ such that

$$
\langle V x, x\rangle \geq \bar{\gamma}\|x\|^{2}, \quad \forall x \in H
$$

A mapping $S: C \rightarrow H$ is called $L$-Lipschitz continuous if there exists a constant $L \geq 0$ such that

$$
\|S x-S y\| \leq L\|x-y\|, \quad \forall x, y \in C .
$$

In particular, if $L=1$, then $S$ is called a nonexpansive mapping; if $L \in[0,1)$, then $S$ is called a contraction.
Let $A: C \rightarrow H$ be a nonlinear mapping on $C$. We consider the following variational inequality problem (VIP): find a point $x \in C$ such that

$$
\langle A x, y-x\rangle \geq 0, \quad \forall y \in C .
$$

The solution set of VIP (3) is denoted by VI $(C, A)$.

Let $\varphi: C \rightarrow \mathbf{R}$ be a real-valued function, $A: H \rightarrow H$ a nonlinear mapping, and $\Theta: C \times C \rightarrow \mathbf{R}$ a bifunction. The generalized mixed equilibrium problem (GMEP) introduced in [1] is to find $x \in C$ such that

$$
\Theta(x, y)+\varphi(y)-\varphi(x)+\langle A x, y-x\rangle \geq 0, \quad \forall y \in C .
$$

We denote the set of solutions of GMEP (4) by $\operatorname{GMEP}(\Theta, \varphi, A)$.

We assume as in [1] that $\Theta: C \times C \rightarrow \mathbf{R}$ is a bifunction satisfying conditions (H1) $-(\mathrm{H} 4)$ and $\varphi: C \rightarrow \mathbf{R}$ is a lower semicontinuous and convex function with restriction (H5), where

(H1) $\Theta(x, x)=0$ for all $x \in C$; 
(H2) $\Theta$ is monotone, that is, $\Theta(x, y)+\Theta(y, x) \leq 0$ for any $x, y \in C$;

(H3) $\Theta$ is upper-hemicontinuous, that is, for each $x, y, z \in$ C,

$$
\limsup _{t \rightarrow 0^{+}} \Theta(t z+(1-t) x, y) \leq \Theta(x, y)
$$

(H4) $\Theta(x, \cdot)$ is convex and lower semicontinuous for each $x \in C$;

(H5) for each $x \in H$ and $r>0$, there exist a bounded subset $D_{x} \subset C$ and $y_{x} \in C$ such that, for any $z \in C \backslash D_{x}$,

$$
\Theta\left(z, y_{x}\right)+\varphi\left(y_{x}\right)-\varphi(z)+\frac{1}{r}\left\langle y_{x}-z, z-x\right\rangle<0 .
$$

Given a positive number, $r>0$. Let $S_{r}^{(\Theta, \varphi)}: H \rightarrow C$ be the solution set of the auxiliary mixed equilibrium problem; that is, for each $x \in H$,

$$
\begin{aligned}
S_{r}^{(\Theta, \varphi)}(x):= & \{y \in C: \Theta(y, z)+\varphi(z)-\varphi(y) \\
& \left.+\frac{1}{r}\left\langle K^{\prime}(y)-K^{\prime}(x), z-y\right\rangle \geq 0, \forall z \in C\right\} .
\end{aligned}
$$

In particular, whenever $K(x)=(1 / 2)\|x\|^{2}$, for all $x \in$ $H, S_{r}^{(\Theta, \varphi)}(x)$ is rewritten as $T_{r}^{(\Theta, \varphi)}(x)$.

Let $\Theta_{1}, \Theta_{2}: C \times C \rightarrow \mathbf{R}$ be two bifunctions and $A_{1}, A_{2}$ : $\mathrm{C} \rightarrow \mathrm{H}$ two nonlinear mappings. Consider the following system of generalized equilibrium problems (SGEP) [2]: find $\left(x^{*}, y^{*}\right) \in C \times C$ such that

$$
\begin{array}{r}
\Theta_{1}\left(x^{*}, x\right)+\left\langle A_{1} y^{*}, x-x^{*}\right\rangle+\frac{1}{v_{1}}\left\langle x^{*}-y^{*}, x-x^{*}\right\rangle \geq 0, \\
\forall x \in C, \\
\Theta_{2}\left(y^{*}, y\right)+\left\langle A_{2} x^{*}, y-y^{*}\right\rangle+\frac{1}{v_{2}}\left\langle y^{*}-x^{*}, y-y^{*}\right\rangle \geq 0, \\
\forall y \in C,
\end{array}
$$

where $v_{1}>0$ and $v_{2}>0$ are two constants.

In 2010, Ceng and Yao [2] transformed the SGEP into a fixed point problem in the following way.

Proposition CY (see [2]). Let $\Theta_{1}, \Theta_{2}: C \times C \rightarrow \mathbf{R}$ be two bifunctions satisfying conditions (H1)-(H4) and let $A_{k}$ : $C \rightarrow H$ be $\zeta_{k}$-inverse-strongly monotone for $k=1,2$. Let $v_{k} \in\left(0,2 \zeta_{k}\right)$ for $k=1,2$. Then, $\left(x^{*}, y^{*}\right) \in C \times C$ is a solution of SGEP (8) if and only if $x^{*}$ is a fixed point of the mapping $G: C \rightarrow C$ defined by $G=T_{\nu_{1}}^{\Theta_{1}}\left(I-\nu_{1} A_{1}\right) T_{\nu_{2}}^{\Theta_{2}}\left(I-\nu_{2} A_{2}\right)$ where $y^{*}=T_{v_{2}}^{\Theta_{2}}\left(I-v_{2} A_{2}\right) x^{*}$. Here, we denote the fixed point set of $G$ by $\operatorname{SGEP}(G)$.
Let $\left\{T_{n}\right\}_{n=1}^{\infty}$ be an infinite family of nonexpansive mappings on $H$ and $\left\{\lambda_{n}\right\}_{n=1}^{\infty}$ a sequence of nonnegative numbers in $[0,1]$. For any $n \geq 1$, define a mapping $W_{n}$ on $H$ as follows:

$$
\begin{aligned}
U_{n, n+1} & =I, \\
U_{n, n} & =\lambda_{n} T_{n} U_{n, n+1}+\left(1-\lambda_{n}\right) I, \\
U_{n, n-1} & =\lambda_{n-1} T_{n-1} U_{n, n}+\left(1-\lambda_{n-1}\right) I, \\
& \vdots \\
U_{n, k} & =\lambda_{k} T_{k} U_{n, k+1}+\left(1-\lambda_{k}\right) I, \\
U_{n, k-1} & =\lambda_{k-1} T_{k-1} U_{n, k}+\left(1-\lambda_{k-1}\right) I, \\
& \vdots \\
U_{n, 2} & =\lambda_{2} T_{2} U_{n, 3}+\left(1-\lambda_{2}\right) I, \\
W_{n} & =U_{n, 1}=\lambda_{1} T_{1} U_{n, 2}+\left(1-\lambda_{1}\right) I .
\end{aligned}
$$

Such a mapping $W_{n}$ is called the $W$-mapping generated by $T_{n}, T_{n-1}, \ldots, T_{1}$ and $\lambda_{n}, \lambda_{n-1}, \ldots, \lambda_{1}$.

Let $Q: H \rightarrow H$ be a contraction and $V$ a strongly positive bounded linear operator on $H$. Assume that $\varphi$ : $H \rightarrow \mathbf{R}$ is a lower semicontinuous and convex functional, that $\Theta, \Theta_{1}, \Theta_{2}: H \times H \rightarrow \mathbf{R}$ satisfy conditions (H1)(H4), and that $A, A_{1}, A_{2}: H \rightarrow H$ are inverse-strongly monotone. Let the mapping $G$ be defined as in Proposition CY. Very recently, Ceng et al. [3] introduced the following hybrid extragradient-like iterative scheme:

$$
\begin{gathered}
z_{n}=S_{r_{n}}^{(\Theta, \varphi)}\left(x_{n}-r_{n} A x_{n}\right), \\
x_{n+1}=\alpha_{n}\left(u+\gamma Q x_{n}\right)+\beta_{n} x_{n} \\
+\left(\left(1-\beta_{n}\right) I-\alpha_{n}(I+\mu V)\right) W_{n} G z_{n}, \quad \forall n \geq 0,
\end{gathered}
$$

for finding a common solution of GMEP (4), SGEP (8), and the fixed point problem of an infinite family of nonexpansive mappings $\left\{T_{n}\right\}_{n=1}^{\infty}$ on $H$, where $\left\{r_{n}\right\} \subset(0, \infty),\left\{\alpha_{n}\right\},\left\{\beta_{n}\right\} \subset$ $(0,1), v_{k} \in\left(0,2 \zeta_{k}\right), k=1,2$, and $x_{0}, u \in H$ are given. The authors proved the strong convergence of the sequence $\left\{x_{n}\right\}$ to a point $x^{*} \in \Omega:=\cap_{n=1}^{\infty} \operatorname{Fix}\left(T_{n}\right) \cap \operatorname{GMEP}(\Theta, \varphi, A) \cap \operatorname{SGEP}(G)$ under some suitable conditions. This point $x^{*}$ also solves the following optimization problem:

$$
\min _{x \in \Omega} \frac{\mu}{2}\langle V x, x\rangle+\frac{1}{2}\|x-u\|^{2}-h(x),
$$

where $h: H \rightarrow \mathbf{R}$ is the potential function of $\gamma Q$.

Let $B$ be a single-valued mapping of $C$ into $H$ and $R$ a multivalued mapping with $D(R)=C$. Consider the following variational inclusion: find a point $x \in C$ such that

$$
0 \in B x+R x \text {. }
$$

We denote by $I(B, R)$ the solution set of the variational inclusion (11). In 1998, Huang [4] studied problem (11) in 
the case where $R$ is maximal monotone and $B$ is strongly monotone and Lipschitz continuous with $D(R)=C=H$.

Let $f: C \rightarrow \mathbf{R}$ be a convex and continuously Fréchet differentiable functional. Consider the convex minimization problem (CMP) of minimizing $f$ over the constraint set $C$ :

$$
\operatorname{minimize}\{f(x): x \in C\} \text {. }
$$

We denote by $\Gamma$ the set of minimizers of CMP (12).

Very recently, Ceng and Al-Homidan [5] introduced an implicit iterative algorithm for finding a common solution of the CMP (12), finitely many GMEPs and finitely many variational inclusions, and derived its strong convergence under appropriate conditions.

Algorithm CA (see [5, Theorem 18]). Let $C$ be a nonempty closed convex subset of a real Hilbert space $H$. Let $f: C \rightarrow \mathbf{R}$ be a convex functional with $L$-Lipschitz continuous gradient $\nabla f$. Let $M, N$ be two integers. Let $\Theta_{k}$ be a bifunction from $C \times C$ to $\mathbf{R}$ satisfying $(\mathrm{H} 1)-(\mathrm{H} 4)$ and $\varphi_{k}: C \rightarrow \mathbf{R} \cup$ $\{+\infty\}$ a proper lower semicontinuous and convex function, where $k \in\{1,2, \ldots, M\}$. Let $R_{i}: C \rightarrow 2^{H}$ be a maximal monotone mapping and let $A_{k}: H \rightarrow H$ and $B_{i}$ : $C \rightarrow H$ be $\mu_{k}$-inverse strongly monotone and $\eta_{i}$-inverse strongly monotone, respectively, where $k \in\{1,2, \ldots, M\}, i \in$ $\{1,2, \ldots, N\}$. Let $F: H \rightarrow H$ be a $\kappa$-Lipschitzian and $\eta$ strongly monotone operator with positive constants $\kappa, \eta>0$. Let $Q: H \rightarrow H$ be an $l$-Lipschitzian mapping with constant $l \geq 0$. Let $0<\mu<\left(2 \eta / \kappa^{2}\right)$ and $0 \leq \gamma l<\tau$, where $\tau=1-\sqrt{1-\mu\left(2 \eta-\mu \kappa^{2}\right)}$. Let $\left\{x_{n}\right\}$ be a sequence generated by

$$
\begin{aligned}
u_{n}= & T_{r_{M, n}}^{\left(\Theta_{M}, \varphi_{M}\right)}\left(I-r_{M, n} A_{M}\right) T_{r_{M-1, n}}^{\left(\Theta_{M-1}, \varphi_{M-1}\right)} \\
& \times\left(I-r_{M-1, n} A_{M-1}\right) \cdots T_{r_{1, n}}^{\left(\Theta_{1}, \varphi_{1}\right)}\left(I-r_{1, n} A_{1}\right) x_{n}, \\
v_{n}= & J_{R_{N}, \lambda_{N, n}}\left(I-\lambda_{N, n} B_{N}\right) J_{R_{N-1}, \lambda_{N-1, n}} \\
& \times\left(I-\lambda_{N-1, n} B_{N-1}\right) \cdots J_{R_{1}, \lambda_{1, n}}\left(I-\lambda_{1, n} B_{1}\right) u_{n}, \\
& x_{n}=s_{n} \gamma Q x_{n}+\left(I-s_{n} \mu F\right) T_{n} v_{n}, \quad \forall n \geq 1,
\end{aligned}
$$

where $P_{C}\left(I-\lambda_{n} \nabla f\right)=s_{n} I+\left(1-s_{n}\right) T_{n}$ (here $T_{n}$ is nonexpansive, $s_{n}=\left(\left(2-\lambda_{n} L\right) / 4\right) \in(0,(1 / 2))$ for each $\left.\lambda_{n} \in(0,(2 / L))\right)$, and the following conditions hold:

(i) $s_{n} \in(0,(1 / 2))$ for each $\lambda_{n} \in(0,(2 / L)), \lim _{n \rightarrow \infty} s_{n}=0$ $\left(\Leftrightarrow \lim _{n \rightarrow \infty} \lambda_{n}=(2 / L)\right)$;

(ii) $\left\{\lambda_{i, n}\right\} \subset\left[a_{i}, b_{i}\right] \subset\left(0,2 \eta_{i}\right)$, for all $i \in\{1,2, \ldots, N\}$;

(iii) $\left\{r_{k, n}\right\} \subset\left[e_{k}, f_{k}\right] \subset\left(0,2 \mu_{k}\right)$, for all $k \in\{1,2, \ldots, M\}$.

Motivated and inspired by the above facts, we first introduce and analyze one implicit iterative algorithm for finding a solution of the CMP (12) with constraints of several problems: the GMEP (4), the SGEP (8), and finitely many variational inclusions in a real Hilbert space. We prove strong convergence theorem for the iterative algorithm under suitable conditions. The iterative algorithm is based on Korpelevich's extragradient method, hybrid steepest-descent method in [6], viscosity approximation method, averaged mapping approach to the GPA in [7], and strongly positive bounded linear operator technique. On the other hand, we also propose another implicit iterative algorithm for finding a fixed point of infinitely many nonexpansive mappings with the same constraints. We derive its strong convergence under mild assumptions.

\section{Preliminaries}

Throughout this paper, we assume that $H$ is a real Hilbert space whose inner product and norm are denoted by $\langle\cdot, \cdot\rangle$ and $\|\cdot\|$, respectively. Let $C$ be a nonempty closed convex subset of $H$. We write $x_{n} \rightarrow x$ to indicate that the sequence $\left\{x_{n}\right\}$ converges weakly to $x$ and $x_{n} \rightarrow x$ to indicate that the sequence $\left\{x_{n}\right\}$ converges strongly to $x$. Moreover, we use $\omega_{w}\left(x_{n}\right)$ to denote the weak $\omega$-limit set of the sequence $\left\{x_{n}\right\}$; that is,

$$
\begin{aligned}
& \omega_{w}\left(x_{n}\right) \\
& :=\left\{x \in H: x_{n_{i}} \rightarrow x \text { for some subsequence }\left\{x_{n_{i}}\right\} \text { of }\left\{x_{n}\right\}\right\} .
\end{aligned}
$$

Recall that a mapping $A: C \rightarrow H$ is called

(i) monotone if

$$
\langle A x-A y, x-y\rangle \geq 0, \quad \forall x, y \in C
$$

(ii) $\eta$-strongly monotone if there exists a constant $\eta>0$ such that

$$
\langle A x-A y, x-y\rangle \geq \eta\|x-y\|^{2}, \quad \forall x, y \in C ;
$$

(iii) $\alpha$-inverse-strongly monotone if there exists a constant $\alpha>0$ such that

$$
\langle A x-A y, x-y\rangle \geq \alpha\|A x-A y\|^{2}, \quad \forall x, y \in C .
$$

It is obvious that if $A$ is $\alpha$-inverse-strongly monotone, then $A$ is monotone and $(1 / \alpha)$-Lipschitz continuous.

The metric (or nearest point) projection from $H$ onto $C$ is the mapping $P_{C}: H \rightarrow C$ which assigns to each point $x \in H$ the unique point $P_{C} x \in C$ satisfying the property

$$
\left\|x-P_{C} x\right\|=\inf _{y \in C}\|x-y\|=: d(x, C) .
$$

Some important properties of projections are gathered in the following proposition.

Proposition 1. For given $x \in H$ and $z \in C$,

(i) $z=P_{C} x \Leftrightarrow\langle x-z, y-z\rangle \leq 0$, for all $y \in C$;

(ii) $z=P_{C} x \Leftrightarrow\|x-z\|^{2} \leq\|x-y\|^{2}-$ $\|y-z\|^{2}$, for all $y \in C$;

(iii) $\left\langle P_{C} x-P_{C} y, x-y\right\rangle \geq\left\|P_{C} x-P_{C} y\right\|^{2}$, for all $y \in H$.

Consequently, $P_{C}$ is nonexpansive and monotone. 
If $A$ is an $\alpha$-inverse-strongly monotone mapping of $C$ into $H$, then it is obvious that $A$ is $(1 / \alpha)$-Lipschitz continuous. If $\lambda \leq 2 \alpha$, then it is easy to see that $I-\lambda A$ is a nonexpansive mapping from $C$ to $H$.

Definition 2. A mapping $T: H \rightarrow H$ is said to be

(a) nonexpansive if

$$
\|T x-T y\| \leq\|x-y\|, \quad \forall x, y \in H
$$

(b) firmly nonexpansive if $2 T-I$ is nonexpansive, or equivalently, if $T$ is 1 -inverse strongly monotone (1ism),

$$
\langle x-y, T x-T y\rangle \geq\|T x-T y\|^{2}, \quad \forall x, y \in H
$$

alternatively, $T$ is firmly nonexpansive if and only if $T$ can be expressed as

$$
T=\frac{1}{2}(I+S)
$$

where $S: H \rightarrow H$ is nonexpansive; projections are firmly nonexpansive.

It can be easily seen that if $T$ is nonexpansive, then $I-T$ is monotone. It is also easy to see that a projection $P_{C}$ is 1 -ism.

Definition 3. A mapping $T: H \rightarrow H$ is said to be an averaged mapping if it can be written as the average of the identity $I$ and a nonexpansive mapping; that is,

$$
T \equiv(1-\alpha) I+\alpha S
$$

where $\alpha \in(0,1)$ and $S: H \rightarrow H$ is nonexpansive. More precisely, when the last equality holds, we say that $T$ is $\alpha$ averaged. Thus firmly nonexpansive mappings (in particular, projections) are (1/2)-averaged mappings.

Proposition 4 (see [8]). Let $T: H \rightarrow H$ be a given mapping.

(i) $T$ is nonexpansive if and only if the complement $I-T$ is $(1 / 2)-i s m$.

(ii) If $T$ is $\nu$-ism, then, for $\gamma>0, \gamma T$ is $(\nu / \gamma)$-ism.

(iii) $T$ is averaged if and only if the complement $I-T$ is $\nu$-ism for some $\nu>1 / 2$. Indeed, for $\alpha \in(0,1), T$ is $\alpha$-averaged if and only if $I-T$ is $(1 / 2 \alpha)$-ism.

Proposition 5 (see [8]). Let $S, T, V: H \rightarrow H$ be given operators.

(i) If $T=(1-\alpha) S+\alpha V$ for some $\alpha \in(0,1)$ and if $S$ is averaged and $V$ is nonexpansive, then $T$ is averaged.

(ii) $T$ is firmly nonexpansive if and only if the complement $I-T$ is firmly nonexpansive.

(iii) If $T=(1-\alpha) S+\alpha V$ for some $\alpha \in(0,1)$ and if $S$ is firmly nonexpansive and $V$ is nonexpansive, then $T$ is averaged. (iv) The composite of finitely many averaged mappings is averaged. That is, if each of the mappings $\left\{T_{i}\right\}_{i=1}^{N}$ is averaged, then so is the composite $T_{1} \cdots T_{N}$. In particular, if $T_{1}$ is $\alpha_{1}$-averaged and $T_{2}$ is $\alpha_{2}$-averaged, where $\alpha_{1}, \alpha_{2} \in(0,1)$, then the composite $T_{1} T_{2}$ is $\alpha$ averaged, where $\alpha=\alpha_{1}+\alpha_{2}-\alpha_{1} \alpha_{2}$.

(v) If the mappings $\left\{T_{i}\right\}_{i=1}^{N}$ are averaged and have a common fixed point, then

$$
\bigcap_{i=1}^{N} \operatorname{Fix}\left(T_{i}\right)=\operatorname{Fix}\left(T_{1} \cdots T_{N}\right) \text {. }
$$

The notation Fix(T) denotes the set of all fixed points of the mapping T; that is, Fix $(T)=\{x \in H: T x=x\}$.

By using the technique in [9], we can readily obtain the following elementary result.

Proposition 6 (see [3, Lemma 1 and Proposition 1]). Let $C$ be a nonempty closed convex subset of a real Hilbert space $H$ and let $\varphi: C \rightarrow \mathbf{R}$ be a lower semicontinuous and convex function. Let $\Theta: C \times C \rightarrow \mathbf{R}$ be a bifunction satisfying the conditions (H1)-(H4). Assume that

(i) $K: H \rightarrow \mathbf{R}$ is strongly convex with constant $\sigma>0$ and the function $x \mapsto\left\langle y-x, K^{\prime}(x)\right\rangle$ is weakly upper semicontinuous for each $y \in H$;

(ii) for each $x \in H$ and $r>0$, there exists a bounded subset $D_{x} \subset C$ and $y_{x} \in C$ such that, for any $z \in C \backslash D_{x}$,

$\Theta\left(z, y_{x}\right)+\varphi\left(y_{x}\right)-\varphi(z)+\frac{1}{r}\left\langle K^{\prime}(z)-K^{\prime}(x), y_{x}-z\right\rangle<0$.

Then the following hold:

(a) for each $x \in H, S_{r}^{(\Theta, \varphi)}(x) \neq \emptyset$;

(b) $S_{r}^{(\Theta, \varphi)}$ is single-valued;

(c) $S_{r}^{(\Theta, \varphi)}$ is nonexpansive if $K^{\prime}$ is Lipschitz continuous with constant $\nu>0$ and

$$
\begin{aligned}
& \left\langle K^{\prime}\left(x_{1}\right)-K^{\prime}\left(x_{2}\right), u_{1}-u_{2}\right\rangle \\
& \quad \leq\left\langle K^{\prime}\left(u_{1}\right)-K^{\prime}\left(u_{2}\right), u_{1}-u_{2}\right\rangle, \quad \forall\left(x_{1}, x_{2}\right) \in H \times H,
\end{aligned}
$$

where $u_{i}=S_{r}^{(\Theta, \varphi)}\left(x_{i}\right)$ for $i=1,2$;

(d) for all $s, t>0$ and $x \in H$

$$
\begin{aligned}
& \left\langle K^{\prime}\left(S_{s}^{(\Theta, \varphi)} x\right)-K^{\prime}\left(S_{t}^{(\Theta, \varphi)} x\right), S_{s}^{(\Theta, \varphi)} x-S_{t}^{(\Theta, \varphi)} x\right\rangle \\
& \quad \leq \frac{s-t}{s}\left\langle K^{\prime}\left(S_{s}^{(\Theta, \varphi)} x\right)-K^{\prime}(x), S_{s}^{(\Theta, \varphi)} x-S_{t}^{(\Theta, \varphi)} x\right\rangle ;
\end{aligned}
$$

(e) $\operatorname{Fix}\left(S_{r}^{(\Theta, \varphi)}\right)=\operatorname{MEP}(\Theta, \varphi)$;

(f) $\operatorname{MEP}(\Theta, \varphi)$ is closed and convex. 
Remark 7. In Proposition 4, whenever $\Theta: C \times C \rightarrow \mathbf{R}$ is a bifunction satisfying the conditions (H1)-(H4) and $K(x)=$ $(1 / 2)\|x\|^{2}$, for all $x \in H$, we have for any $x, y \in H$,

$$
\left\|S_{r}^{(\Theta, \varphi)} x-S_{r}^{(\Theta, \varphi)} y\right\|^{2} \leq\left\langle S_{r}^{(\Theta, \varphi)} x-S_{r}^{(\Theta, \varphi)} y, x-y\right\rangle
$$

$\left(S_{r}^{(\Theta, \varphi)}\right.$ is firmly nonexpansive $)$ and

$$
\begin{array}{r}
\left\|S_{s}^{(\Theta, \varphi)} x-S_{t}^{(\Theta, \varphi)} x\right\| \leq \frac{|s-t|}{s}\left\|S_{s}^{(\Theta, \varphi)} x-x\right\|, \\
\forall s, t>0, x \in H .
\end{array}
$$

In this case, $S_{r}^{(\Theta, \varphi)}$ is rewritten as $T_{r}^{(\Theta, \varphi)}$. If, in addition, $\varphi \equiv 0$, then $T_{r}^{(\Theta, \varphi)}$ is rewritten as $T_{r}^{\Theta}$; see [2, Lemma 2.1] for more details.

We need some facts and tools in a real Hilbert space $H$ which are listed as lemmas below.

Lemma 8. Let $X$ be a real inner product space. Then there holds the following inequality:

$$
\|x+y\|^{2} \leq\|x\|^{2}+2\langle y, x+y\rangle, \quad \forall x, y \in X .
$$

Lemma 9. Let $A: C \rightarrow H$ be a monotone mapping. In the context of the variational inequality problem the characterization of the projection (see Proposition 1(i)) implies

$$
u \in V I(C, A) \Longleftrightarrow u=P_{C}(u-\lambda A u), \quad \lambda>0 .
$$

Lemma 10 (see [10, demiclosedness principle]). Let $C$ be a nonempty closed convex subset of a real Hilbert space $H$. Let $T$ be a nonexpansive self-mapping on $C$. Then $I-T$ is demiclosed. That is, whenever $\left\{x_{n}\right\}$ is a sequence in $C$ weakly converging to some $x \in C$ and the sequence $\left\{(I-T) x_{n}\right\}$ strongly converges to some $y$, it follows that $(I-T) x=y$. Here $I$ is the identity operator of $H$.

Lemma 11 (see [11, Lemma 3.2]). Let $C$ be a nonempty closed convex subset of a real Hilbert space $H$. Let $\left\{T_{n}\right\}_{n=1}^{\infty}$ be a sequence of nonexpansive self-mappings on $C$ such that $\cap_{n=1}^{\infty}$ Fix $\left(T_{n}\right) \neq \emptyset$ and let $\left\{\lambda_{n}\right\}$ be a sequence in $(0, b]$ for some $b \in(0,1)$. Then, for every $x \in C$ and $k \geq 1$ the limit $\lim _{n \rightarrow \infty} U_{n, k} x$ exists where $U_{n, k}$ is defined as in (9).

Lemma 12 (see [11, Lemma 3.3]). Let $C$ be a nonempty closed convex subset of a real Hilbert space $H$. Let $\left\{T_{n}\right\}_{n=1}^{\infty}$ be a sequence of nonexpansive self-mappings on $C$ such that $\cap_{n=1}^{\infty} F i x\left(T_{n}\right) \neq \emptyset$, and let $\left\{\lambda_{n}\right\}$ be a sequence in $(0, b]$ for some $b \in(0,1)$. Then, Fix $(W)=\cap_{n=1}^{\infty} \operatorname{Fix}\left(T_{n}\right)$.

The following lemma can be easily proved and, therefore, we omit the proof.

Lemma 13. Let $V: H \rightarrow H$ be a $\bar{\gamma}$-strongly positive bounded linear operator with constant $\bar{\gamma}>1$. Then for $\bar{\gamma}-1>0$,

$$
\begin{array}{r}
\langle(V-I) x-(V-I) y, x-y\rangle \geq(\bar{\gamma}-1)\|x-y\|^{2}, \\
\forall x, y \in H .
\end{array}
$$

That is, $V-I$ is strongly monotone with constant $\bar{\gamma}-1$.
Let $C$ be a nonempty closed convex subset of a real Hilbert space $H$. We introduce some notations. Let $\lambda$ be a number in $(0,1]$ and let $\mu>0$. Associating with a nonexpansive mapping $T: C \rightarrow H$, we define the mapping $T^{\lambda}: C \rightarrow H$ by

$$
T^{\lambda} x:=T x-\lambda \mu F(T x), \quad \forall x \in C,
$$

where $F: H \rightarrow H$ is an operator such that, for some positive constants $\kappa, \eta>0, F$ is $\kappa$-Lipschitzian and $\eta$-strongly monotone on $H$; that is, $F$ satisfies the conditions

$$
\|F x-F y\| \leq \kappa\|x-y\|, \quad\langle F x-F y, x-y\rangle \geq \eta\|x-y\|^{2}
$$

for all $x, y \in H$.

Lemma 14 (see [12, Lemma 3.1]). $T^{\lambda}$ is a contraction provided $0<\mu<\left(2 \eta / \kappa^{2}\right)$; that is,

$$
\left\|T^{\lambda} x-T^{\lambda} y\right\| \leq(1-\lambda \tau)\|x-y\|, \quad \forall x, y \in C,
$$

where $\tau=1-\sqrt{1-\mu\left(2 \eta-\mu \kappa^{2}\right)} \in(0,1]$.

We observe that since $F$ is $\kappa$-Lipschitzian and $\eta$-strongly monotone on $H$, we get $0<\eta \leq \kappa$. Hence, whenever $0<$ $\mu<\left(2 \eta / \kappa^{2}\right)$, we have $\tau=1-\sqrt{1-\mu\left(2 \eta-\mu \kappa^{2}\right)} \in(0,1]$. Also in Lemma 14 , put $F=(1 / 2) I$ and $\mu=2$. Then we know that $\kappa=\eta=(1 / 2), 0<\mu=2<\left(2 \eta / \kappa^{2}\right)=4$ and $\tau=1$.

Recall that a set-valued mapping $R: D(R) \subset H \rightarrow 2^{H}$ is called monotone if for all $x, y \in D(R), f \in R(x)$ and $g \in R(y)$ imply

$$
\langle f-g, x-y\rangle \geq 0 \text {. }
$$

A set-valued mapping $R$ is called maximal monotone if $R$ is monotone and $(I+\lambda R) D(R)=H$ for each $\lambda>0$, where $I$ is the identity mapping of $H$. We denote by $G(R)$ the graph of $R$. It is known that a monotone mapping $R$ is maximal if and only if, for $(x, f) \in H \times H,\langle f-g, x-y\rangle \geq 0$ for every $(y, g) \in G(R)$ implies $f \in R(x)$.

Let $A: C \rightarrow H$ be a monotone, $k$-Lipschitz-continuous mapping and let $N_{C} v$ be the normal cone to $C$ at $v \in C$; that is,

$$
N_{C} v=\{w \in H:\langle v-u, w\rangle \geq 0, \forall u \in C\}
$$

Define

$$
T v= \begin{cases}A v+N_{C} v, & \text { if } v \in C, \\ \emptyset, & \text { if } v \notin C .\end{cases}
$$

It is well known that $T$ is maximal monotone and $0 \in T v$ if and only if $v \in V I(C, A)$.

Assume that $R: D(R) \subset H \rightarrow 2^{H}$ is a maximal monotone mapping. Then, for $\lambda>0$, associated with $R$, the resolvent operator $J_{R, \lambda}$ can be defined as

$$
J_{R, \lambda} x=(I+\lambda R)^{-1} x, \quad \forall x \in H .
$$

From Huang [4] (see also [13]), there holds the following property for the resolvent operator $J_{R, \lambda}: H \rightarrow \overline{D(R)}$. 
Lemma 15. $J_{R, \lambda}$ is single-valued and firmly nonexpansive; that is,

$$
\left\langle J_{R, \lambda} x-J_{R, \lambda} y, x-y\right\rangle \geq\left\|J_{R, \lambda} x-J_{R, \lambda} y\right\|^{2}, \quad \forall x, y \in H .
$$

Consequently, $J_{R, \lambda}$ is nonexpansive and monotone.

Lemma 16 (see [14]). Let $R$ be a maximal monotone mapping with $D(R)=C$. Then for any given $\lambda>0, u \in C$ is a solution of problem (11) if and only if $u \in C$ satisfies

$$
u=J_{R, \lambda}(u-\lambda B u) .
$$

Lemma 17 (see [13]). Let $R$ be a maximal monotone mapping with $D(R)=C$ and let $B: C \rightarrow H$ be a strongly monotone, continuous, and single-valued mapping. Then for each $z \in H$, the equation $z \in(B+\lambda R) x$ has a unique solution $x_{\lambda}$ for $\lambda>0$.

Lemma 18 (see [14]). Let $R$ be a maximal monotone mapping with $D(R)=C$ and $B: C \rightarrow H$ a monotone, continuous, and single-valued mapping. Then $(I+\lambda(R+B)) C=H$ for each $\lambda>0$. In this case, $R+B$ is maximal monotone.

\section{Convex Minimization Problems with Constraints}

In this section, we will introduce and analyze one implicit iterative algorithm for finding a solution of the CMP (12) with constraints of several problems: the GMEP (4), the SGEP (8), and finitely many variational inclusions in a real Hilbert space. We po prove strong convergence theorem for the iterative algorithm under suitable conditions.

Theorem 19. Let $C$ be a nonempty closed convex subset of a real Hilbert space $H$. Let $N$ be an integer. Let $f: C \rightarrow \mathbf{R}$ be a convex functional with L-Lipschitz continuous gradient $\nabla f$. Let $\Theta, \Theta_{1}, \Theta_{2}$ be three bifunctions from $C \times C$ to $\mathbf{R}$ satisfying (H1)-(H4) and $\varphi: C \rightarrow \mathbf{R}$ a lower semicontinuous and convex functional. Let $R_{i}: C \rightarrow 2^{H}$ be a maximal monotone mapping and let $A: H \rightarrow H$ and let $A_{k}, B_{i}: C \rightarrow H$ be $\zeta$-inverse strongly monotone, $\zeta_{k}$-inverse strongly monotone, and $\eta_{i}$-inverse-strongly monotone, respectively, for $k=1,2$ and $i=1,2, \ldots, N$. Let $V$ be a $\bar{\gamma}$-strongly positive bounded linear operator with $\bar{\gamma}>1$. Let $F: H \rightarrow H$ be $a \kappa-$ Lipschitzian and $\eta$-strongly monotone operator with positive constants $\kappa, \eta>0$. Let $Q: H \rightarrow H$ be an l-Lipschitzian mapping with constant $l \geq 0$. Let $0<\mu<\left(2 \eta / \kappa^{2}\right)$ and $0 \leq \gamma l<\tau$, where $\tau=1-\sqrt{1-\mu\left(2 \eta-\mu \kappa^{2}\right)}$. Assume that $\Omega:=\operatorname{GMEP}(\Theta, \varphi, A) \cap S G E P(G) \cap \cap_{i=1}^{N} I\left(B_{i}, R_{i}\right) \cap \Gamma \neq \emptyset$ where $G$ is defined as in Proposition CY. Let $\left\{r_{n}\right\}$ be a sequence in $(0,2 \zeta]$ and $\left\{\alpha_{n}\right\}$ a sequence in $(0,1]$ such that $\lim _{n \rightarrow \infty} \alpha_{n}=0$. Let $\left\{x_{n}\right\}$ be the sequence generated by

$$
\begin{gathered}
u_{n}=S_{r_{n}}^{(\Theta, \varphi)}\left(I-r_{n} A\right) x_{n}, \\
v_{n}=J_{R_{N}, \lambda_{N, n}}\left(I-\lambda_{N, n} B_{N}\right) J_{R_{N-1}, \lambda_{N-1, n}} \\
\times\left(I-\lambda_{N-1, n} B_{N-1}\right) \cdots J_{R_{1}, \lambda_{1, n}}\left(I-\lambda_{1, n} B_{1}\right) u_{n},
\end{gathered}
$$

$$
\begin{aligned}
x_{n}= & \left(I-s_{n} V\right) T_{n} G v_{n} \\
& +s_{n}\left[T_{n} x_{n}-\alpha_{n}\left(\mu F\left(T_{n} x_{n}\right)-\gamma Q x_{n}\right)\right], \quad \forall n \geq 1,
\end{aligned}
$$

where $P_{C}\left(I-\lambda_{n} \nabla f\right)=s_{n} I+\left(1-s_{n}\right) T_{n}$ (here $T_{n}$ is nonexpansive, $s_{n}=\left(\left(2-\lambda_{n} L\right) / 4\right) \in(0,(1 / 2))$ for each $\left.\lambda_{n} \in(0,(2 / L))\right)$. Suppose that the following conditions hold.

(i) $\mathrm{K}: H \rightarrow \mathbf{R}$ is strongly convex with constant $\sigma>0$ and its derivative $K^{\prime}$ is Lipschitz continuous with constant $v>0$ such that the function $x \mapsto\left\langle y-x, K^{\prime}(x)\right\rangle$ is weakly upper semicontinuous for each $y \in H$;

(ii) for each $x \in H$, there exist a bounded subset $D_{x} \subset C$ and $z_{x} \in C$ such that, for any $y \notin D_{x}$,

$\Theta\left(y, z_{x}\right)+\varphi\left(z_{x}\right)-\varphi(y)+\frac{1}{r}\left\langle K^{\prime}(y)-K^{\prime}(x), z_{x}-y\right\rangle<0 ;$

(iii) $s_{n} \in(0,(1 / 2))$ for each $\lambda_{n} \in(0,(2 / L)), \lim _{n \rightarrow \infty} s_{n}=$ $0\left(\Leftrightarrow \lim _{n \rightarrow \infty} \lambda_{n}=(2 / L)\right) ;$

(iv) $\nu_{k} \in\left(0,2 \zeta_{k}\right), k=1,2$ and $\left\{\lambda_{i, n}\right\} \subset\left[a_{i}, b_{i}\right] \subset$ $\left(0,2 \eta_{i}\right), i=1,2, \ldots, N$;

(v) $0<\liminf _{n \rightarrow \infty} r_{n} \leq \lim \sup _{n \rightarrow \infty} r_{n}<2 \zeta$.

Assume that $S_{r}^{(\Theta, \varphi)}$ is firmly nonexpansive. Then $\left\{x_{n}\right\}$ converges strongly as $\lambda_{n} \rightarrow(2 / L)\left(\Leftrightarrow s_{n} \rightarrow 0\right)$ to a point $q \in \Omega$, which is a unique solution in $\Omega$ to the VIP:

$$
\langle(I-V) q, p-q\rangle \leq 0, \quad \forall p \in \Omega .
$$

Proof. First of all, let us show that the sequence $\left\{x_{n}\right\}$ is well defined. Indeed, since $\nabla f$ is $L$-Lipschitzian, it follows that $\nabla f$ is $1 / L$-ism. By Proposition 4(ii) we know that, for $\lambda>$ $0, \lambda \nabla f$ is $(1 / \lambda L)$-ism. So by Proposition 4 (iii) we deduce that $I-\lambda \nabla f$ is $(\lambda L / 2)$-averaged. Now since the projection $P_{C}$ is $(1 / 2)$-averaged, it is easy to see from Proposition 5(iv) that the composite $P_{C}(I-\lambda \nabla f)$ is $((2+\lambda L) / 4)$-averaged for $\lambda \epsilon$ $(0,(2 / L))$. Hence we obtain that, for each $n \geq 1, P_{C}\left(I-\lambda_{n} \nabla f\right)$ is $\left(\left(2+\lambda_{n} L\right) / 4\right)$-averaged for each $\lambda_{n} \in(0,(2 / L))$. Therefore, we can write

$$
P_{C}\left(I-\lambda_{n} \nabla f\right)=\frac{2-\lambda_{n} L}{4} I+\frac{2+\lambda_{n} L}{4} T_{n}=s_{n} I+\left(1-s_{n}\right) T_{n},
$$

where $T_{n}$ is nonexpansive and $s_{n}:=s_{n}\left(\lambda_{n}\right)=\left(\left(2-\lambda_{n} L\right) / 4\right) \epsilon$ $(0,(1 / 2))$ for each $\lambda_{n} \in(0,(2 / L))$. It is clear that

$$
\lambda_{n} \longrightarrow \frac{2}{L} \Longleftrightarrow s_{n} \longrightarrow 0 .
$$

As $\lim _{n \rightarrow \infty} s_{n}=0$ and $0<\liminf _{n \rightarrow \infty} r_{n} \leq \limsup _{n \rightarrow \infty} r_{n}<$ $2 \zeta$, we may assume, without loss of generality, that $\left\{r_{n}\right\} \subset$ $[a, \hat{a}] \subset(0,2 \zeta)$ and $s_{n}\|V\| \leq 1$ for all $n \geq 1$. Since $V$ is a $\bar{\gamma}$-strongly positive bounded linear operator on $H$, we know that

$$
\|V\|=\sup \{\langle V u, u\rangle: u \in H,\|u\|=1\} \geq \bar{\gamma}>1 .
$$


Taking into account that $s_{n}\|V\| \leq 1$ for all $n \geq 1$, we have

$$
\left\langle\left(I-s_{n} V\right) u, u\right\rangle=1-s_{n}\langle V u, u\rangle \geq 1-s_{n}\|V\| \geq 0,
$$

that is, $\left(1-\beta_{n}\right) I-s_{n} V$ is positive. It follows that

$$
\begin{aligned}
\left\|I-s_{n} V\right\| & =\sup \left\{\left\langle\left(I-s_{n} V\right) u, u\right\rangle: u \in H,\|u\|=1\right\} \\
& =\sup \left\{1-s_{n}\langle V u, u\rangle: u \in H,\|u\|=1\right\} \\
& \leq 1-s_{n} \bar{\gamma} .
\end{aligned}
$$

Put

$$
\begin{aligned}
\Lambda_{n}^{i}= & J_{R_{i}, \lambda_{i, n}}\left(I-\lambda_{i, n} B_{i}\right) J_{R_{i-1}, \lambda_{i-1, n}} \\
& \times\left(I-\lambda_{i-1, n} B_{i-1}\right) \cdots J_{R_{1}, \lambda_{1, n}}\left(I-\lambda_{1, n} B_{1}\right)
\end{aligned}
$$

for all $i \in\{1,2, \ldots, N\}$, and $\Lambda_{n}^{0}=I$, where $I$ is the identity mapping on $H$. Then we have $v_{n}=\Lambda_{n}^{N} u_{n}$.

Consider the following mapping $G_{n}$ on $H$ defined by

$$
\begin{aligned}
G_{n} x= & \left(I-s_{n} V\right) T_{n} G \Lambda_{n}^{N} S_{r_{n}}^{(\Theta, \varphi)}\left(I-r_{n} A\right) x \\
& +s_{n}\left[T_{n} x-\alpha_{n}\left(\mu F\left(T_{n} x\right)-\gamma Q x\right)\right], \quad \forall x \in H, n \geq 1,
\end{aligned}
$$

where $s_{n}=\left(\left(2-\lambda_{n} L\right) / 4\right) \in(0,(1 / 2))$ for each $\lambda_{n} \in(0,(2 / L))$. Since $A_{k}$ is $\zeta_{k}$-inverse-strongly monotone with $0 \leq \nu_{k} \leq 2 \zeta_{k}$ for $k=1,2$, we deduce that. for any $x, y \in H$,

$$
\begin{aligned}
& \|G x-G y\|^{2} \\
& =\| T_{v_{1}}^{\Theta_{1}}\left(I-v_{1} A_{1}\right) T_{v_{2}}^{\Theta_{2}}\left(I-v_{2} A_{2}\right) x \\
& -T_{v_{1}}^{\Theta_{1}}\left(I-v_{1} A_{1}\right) T_{v_{2}}^{\Theta_{2}}\left(I-v_{2} A_{2}\right) y \|^{2} \\
& \leq \|\left(I-v_{1} A_{1}\right) T_{v_{2}}^{\Theta_{2}}\left(I-v_{2} A_{2}\right) x \\
& -\left(I-v_{1} A_{1}\right) T_{v_{2}}^{\Theta_{2}}\left(I-v_{2} A_{2}\right) y \|^{2} \\
& =\|\left[T_{v_{2}}^{\Theta_{2}}\left(I-v_{2} A_{2}\right) x-T_{v_{2}}^{\Theta_{2}}\left(I-v_{2} A_{2}\right) y\right] \\
& -v_{1}\left[A_{1} T_{v_{2}}^{\Theta_{2}}\left(I-v_{2} A_{2}\right) x-A_{1} T_{v_{2}}^{\Theta_{2}}\left(I-v_{2} A_{2}\right) y\right] \|^{2} \\
& \leq\left\|T_{\nu_{2}}^{\Theta_{2}}\left(I-v_{2} A_{2}\right) x-T_{v_{2}}^{\Theta_{2}}\left(I-v_{2} A_{2}\right) y\right\|^{2}+v_{1}\left(\nu_{1}-2 \zeta_{1}\right) \\
& \times\left\|A_{1} T_{v_{2}}^{\Theta_{2}}\left(I-v_{2} A_{2}\right) x-A_{1} T_{v_{2}}^{\Theta_{2}}\left(I-v_{2} A_{2}\right) y\right\|^{2} \\
& \leq\left\|T_{v_{2}}^{\Theta_{2}}\left(I-v_{2} A_{2}\right) x-T_{v_{2}}^{\Theta_{2}}\left(I-v_{2} A_{2}\right) y\right\|^{2} \\
& \leq\left\|\left(I-v_{2} A_{2}\right) x-\left(I-v_{2} A_{2}\right) y\right\|^{2} \\
& =\left\|(x-y)-v_{2}\left(A_{2} x-A_{2} y\right)\right\|^{2} \\
& \leq\|x-y\|^{2}+v_{2}\left(\nu_{2}-2 \zeta_{2}\right)\left\|A_{2} x-A_{2} y\right\|^{2} \\
& \leq\|x-y\|^{2} \text {. }
\end{aligned}
$$

By the nonexpansivity of $S_{r}^{(\Theta, \varphi)}$ and Lemma 14 we obtain from (41), (48), and (51) that for all $x, y \in C$

$$
\begin{aligned}
& \left\|G_{n} x-G_{n} y\right\| \\
& \leq \|\left(I-s_{n} V\right)\left(T_{n} G \Lambda_{n}^{N} S_{r_{n}}^{(\Theta, \varphi)}\left(I-r_{n} A\right)\right) x \\
& -T_{n} G \Lambda_{n}^{N} S_{r_{n}}^{(\Theta, \varphi)}\left(I-r_{n} A\right) y \| \\
& +s_{n}\left\|\left(I-\alpha_{n} \mu F\right) T_{n} x-\left(I-\alpha_{n} \mu F\right) T_{n} y\right\| \\
& +s_{n} \alpha_{n} \gamma\|Q x-Q y\| \\
& \leq\left(1-s_{n} \bar{\gamma}\right) \\
& \times \| T_{n} G \Lambda_{n}^{N} S_{r_{n}}^{(\Theta, \varphi)}\left(I-r_{n} A\right) x \\
& -T_{n} G \Lambda_{n}^{N} S_{r_{n}}^{(\Theta, \varphi)}\left(I-r_{n} A\right) y \| \\
& +s_{n}\left(1-\alpha_{n} \tau\right)\|x-y\|+s_{n} \alpha_{n} \gamma l\|x-y\| \\
& \leq\left(1-s_{n} \bar{\gamma}\right) \\
& \times\left\|G \Lambda_{n}^{N} S_{r_{n}}^{(\Theta, \varphi)}\left(I-r_{n} A\right) x-G \Lambda_{n}^{N} S_{r_{n}}^{(\Theta, \varphi)}\left(I-r_{n} A\right) y\right\| \\
& +s_{n}\left(1-\alpha_{n} \tau\right)\|x-y\|+s_{n} \alpha_{n} \gamma l\|x-y\| \\
& \leq\left(1-s_{n} \bar{\gamma}\right) \\
& \times\left\|\Lambda_{n}^{N} S_{r_{n}}^{(\Theta, \varphi)}\left(I-r_{n} A\right) x-\Lambda_{n}^{N} S_{r_{n}}^{(\Theta, \varphi)}\left(I-r_{n} A\right) y\right\| \\
& +s_{n}\left(1-\alpha_{n} \tau\right)\|x-y\|+s_{n} \alpha_{n} \gamma l\|x-y\| \\
& \leq\left(1-s_{n} \bar{\gamma}\right) \\
& \times \|\left(I-\lambda_{N, n} B_{N}\right) \Lambda_{n}^{N-1} S_{r_{n}}^{(\Theta, \varphi)}\left(I-r_{n} A\right) x \\
& -\left(I-\lambda_{N, n} B_{N}\right) \Lambda_{n}^{N-1} S_{r_{n}}^{(\Theta, \varphi)}\left(I-r_{n} A\right) y \| \\
& +s_{n}\left(1-\alpha_{n} \tau\right)\|x-y\|+s_{n} \alpha_{n} \gamma l\|x-y\| \\
& \leq\left(1-s_{n} \bar{\gamma}\right) \\
& \times\left\|\Lambda_{n}^{N-1} S_{r_{n}}^{(\Theta, \varphi)}\left(I-r_{n} A\right) x-\Lambda_{n}^{N-1} S_{r_{n}}^{(\Theta, \varphi)}\left(I-r_{n} A\right) y\right\| \\
& +s_{n}\left(1-\alpha_{n} \tau\right)\|x-y\|+s_{n} \alpha_{n} \gamma l\|x-y\| \\
& \leq\left(1-s_{n} \bar{\gamma}\right)\left\|\Lambda_{n}^{0} S_{r_{n}}^{(\Theta, \varphi)}\left(I-r_{n} A\right) x-\Lambda_{n}^{0} S_{r_{n}}^{(\Theta, \varphi)}\left(I-r_{n} A\right) y\right\| \\
& +s_{n}\left(1-\alpha_{n} \tau\right)\|x-y\|+s_{n} \alpha_{n} \gamma l\|x-y\| \\
& =\left(1-s_{n} \bar{\gamma}\right)\left\|S_{r_{n}}^{(\Theta, \varphi)}\left(I-r_{n} A\right) x-S_{r_{n}}^{(\Theta, \varphi)}\left(I-r_{n} A\right) y\right\| \\
& +s_{n}\left(1-\alpha_{n} \tau\right)\|x-y\|+s_{n} \alpha_{n} \gamma l\|x-y\| \\
& \leq\left(1-s_{n} \bar{\gamma}\right)\left\|\left(I-r_{n} A\right) x-\left(I-r_{n} A\right) y\right\| \\
& +s_{n}\left(1-\alpha_{n} \tau\right)\|x-y\|+s_{n} \alpha_{n} \gamma l\|x-y\|
\end{aligned}
$$




$$
\begin{aligned}
\leq & \left(1-s_{n} \bar{\gamma}\right)\|x-y\|+s_{n}\left(1-\alpha_{n} \tau\right)\|x-y\| \\
& +s_{n} \alpha_{n} \gamma l\|x-y\| \\
\leq & \left(1-s_{n}\right)\|x-y\| \\
& +s_{n}\left(1-\alpha_{n} \tau\right)\|x-y\|+s_{n} \alpha_{n} \gamma l\|x-y\| \\
= & \left(1-s_{n} \alpha_{n}(\tau-\gamma l)\right)\|x-y\| .
\end{aligned}
$$

Since $0<1-s_{n} \alpha_{n}(\tau-\gamma l)<1, G_{n}: H \rightarrow H$ is a contraction. Therefore, by the Banach contraction principle, $G_{n}$ has a unique fixed point $x_{n} \in H$, which uniquely solves the fixed point equation

$$
x_{n}=\left(I-s_{n} V\right) T_{n} G v_{n}+s_{n}\left[T_{n} x_{n}-\alpha_{n}\left(\mu F\left(T_{n} x_{n}\right)-\gamma Q x_{n}\right)\right] .
$$

This shows that the sequence $\left\{x_{n}\right\}$ is defined well.

$$
\text { Note that }
$$

$$
\begin{array}{r}
\langle(V-I) x-(V-I) y, x-y\rangle \geq(\bar{\gamma}-1)\|x-y\|^{2}, \\
\forall x, y \in H .
\end{array}
$$

That is, $V-I$ is $(\bar{\gamma}-1)$-strongly monotone for $\bar{\gamma}-1>0$. Moreover, it is clear that $V-I$ is Lipschitz continuous. So the VIP (43) has only one solution in $\Omega$. Below we use $q \in \Omega$ to denote the unique solution of the VIP (43).

Now, let us show that $\left\{x_{n}\right\}$ is bounded. In fact, take $p \in \Omega$ arbitrarily. Then from the nonexpansivity of $S_{r}^{(\Theta, \varphi)}$ and $\left\{r_{n}\right\} \subset$ $[a, \widehat{a}] \subset(0,2 \zeta)$ we have

$$
\begin{aligned}
\| u_{n}- & p \|^{2} \\
= & \left\|S_{r_{n}}^{(\Theta, \varphi)}\left(I-r_{n} A\right) x_{n}-S_{r_{n}}^{(\Theta, \varphi)}\left(I-r_{n} A\right) p\right\|^{2} \\
\leq & \left\|\left(I-r_{n} A\right) x_{n}-\left(I-r_{n} A\right) p\right\|^{2} \\
= & \left\|\left(x_{n}-p\right)-r_{n}\left(A x_{n}-A p\right)\right\|^{2} \\
= & \left\|x_{n}-p\right\|^{2}-2 r_{n}\left\langle x_{n}-p, A x_{n}-A p\right\rangle \\
& +r_{n}^{2}\left\|A x_{n}-A p\right\|^{2} \leq\left\|x_{n}-p\right\|^{2} \\
& -2 r_{n} \zeta\left\|A x_{n}-A p\right\|^{2}+r_{n}^{2}\left\|A x_{n}-A p\right\|^{2} \\
= & \left\|x_{n}-p\right\|^{2}+r_{n}\left(r_{n}-2 \zeta\right)\left\|A x_{n}-A p\right\|^{2} \\
\leq & \left\|x_{n}-p\right\|^{2} .
\end{aligned}
$$

Since $p=J_{R_{i}, \lambda_{i, n}}\left(I-\lambda_{i, n} B_{i}\right) p, \Lambda_{n}^{i} p=p$ and $B_{i}$ is $\eta_{i}$-inverse strongly monotone, where $\lambda_{i, n} \in\left(0,2 \eta_{i}\right), i \in\{1,2, \ldots, N\}$, by Lemma 15 we deduce that for each $n \geq 1$

$$
\begin{aligned}
\left\|v_{n}-p\right\|^{2}= & \left\|\Lambda_{n}^{N} u_{n}-p\right\|^{2} \\
\leq & \left\|\Lambda_{n}^{i} u_{n}-p\right\|^{2} \\
= & \left\|J_{R_{i}, \lambda_{i, n}}\left(I-\lambda_{i, n} B_{i}\right) \Lambda_{n}^{i-1} u_{n}-J_{R_{i}, \lambda_{i, n}}\left(I-\lambda_{i, n} B_{i}\right) p\right\|^{2} \\
\leq & \left\|\left(I-\lambda_{i, n} B_{i}\right) \Lambda_{n}^{i-1} u_{n}-\left(I-\lambda_{i, n} B_{i}\right) p\right\|^{2} \\
\leq & \left\|\Lambda_{n}^{i-1} u_{n}-p\right\|^{2}+\lambda_{i, n}\left(\lambda_{i, n}-2 \eta_{i}\right) \\
& \times\left\|B_{i} \Lambda_{n}^{i-1} u_{n}-B_{i} p\right\|^{2} \\
\leq & \left\|u_{n}-p\right\|^{2} \\
& +\lambda_{i, n}\left(\lambda_{i, n}-2 \eta_{i}\right)\left\|B_{i} \Lambda_{n}^{i-1} u_{n}-B_{i} p\right\|^{2} \\
\leq & \left\|u_{n}-p\right\|^{2} .
\end{aligned}
$$

Combining (55) and (56), we have

$$
\left\|v_{n}-p\right\| \leq\left\|x_{n}-p\right\|
$$

Since $p=G p=T_{v_{1}}^{\Theta_{1}}\left(I-\nu_{1} A_{1}\right) T_{v_{2}}^{\Theta_{2}}\left(I-v_{2} A_{2}\right) p, A_{k}$ is $\zeta_{k^{-}}$ inverse-strongly monotone for $k=1,2$, and $0 \leq \nu_{k} \leq 2 \zeta_{k}$ for $k=1,2$, we deduce that, for any $n \geq 1$,

$$
\begin{aligned}
\left\|G v_{n}-p\right\|^{2} & \| T_{v_{1}}^{\Theta_{1}}\left(I-v_{1} A_{1}\right) T_{v_{2}}^{\Theta_{2}}\left(I-v_{2} A_{2}\right) v_{n} \\
& \quad-T_{v_{1}}^{\Theta_{1}}\left(I-v_{1} A_{1}\right) T_{v_{2}}^{\Theta_{2}}\left(I-v_{2} A_{2}\right) p \|^{2} \\
\leq & \|\left(I-v_{1} A_{1}\right) T_{v_{2}}^{\Theta_{2}}\left(I-v_{2} A_{2}\right) v_{n} \\
& \quad-\left(I-v_{1} A_{1}\right) T_{v_{2}}^{\Theta_{2}}\left(I-v_{2} A_{2}\right) p \|^{2} \\
= & \|\left[T_{v_{2}}^{\Theta_{2}}\left(I-v_{2} A_{2}\right) v_{n}-T_{v_{2}}^{\Theta_{2}}\left(I-v_{2} A_{2}\right) p\right] \\
& \quad-v_{1}\left[A_{1} T_{v_{2}}^{\Theta_{2}}\left(I-v_{2} A_{2}\right) v_{n}-A_{1} T_{v_{2}}^{\Theta_{2}}\left(I-v_{2} A_{2}\right) p\right] \|^{2} \\
\leq & \left\|T_{v_{2}}^{\Theta_{2}}\left(I-v_{2} A_{2}\right) v_{n}-T_{v_{2}}^{\Theta_{2}}\left(I-v_{2} A_{2}\right) p\right\|^{2}+v_{1}\left(v_{1}-2 \zeta_{1}\right) \\
& \times\left\|A_{1} T_{v_{2}}^{\Theta_{2}}\left(I-v_{2} A_{2}\right) v_{n}-A_{1} T_{v_{2}}^{\Theta_{2}}\left(I-v_{2} A_{2}\right) p\right\|^{2} \\
\leq & \left\|T_{v_{2}}^{\Theta_{2}}\left(I-v_{2} A_{2}\right) v_{n}-T_{v_{2}}^{\Theta_{2}}\left(I-v_{2} A_{2}\right) p\right\|^{2} \\
\leq & \left\|\left(I-v_{2} A_{2}\right) v_{n}-\left(I-v_{2} A_{2}\right) p\right\|^{2} \\
= & \left\|\left(v_{n}-p\right)-v_{2}\left(A_{2} v_{n}-A_{2} p\right)\right\|^{2} \\
\leq & \left\|v_{n}-p\right\|^{2}+v_{2}\left(v_{2}-2 \zeta_{2}\right)\left\|A_{2} v_{n}-A_{2} p\right\|^{2} \\
\leq &
\end{aligned}
$$


Thus, utilizing Lemmas 8 and 14, from (41), (43), (55), (56), and (58) we have

$$
\begin{aligned}
& \left\|x_{n}-p\right\|^{2} \\
& =\|\left(I-s_{n} V\right)\left(T_{n} G v_{n}-p\right) \\
& +s_{n}\left[T_{n} x_{n}-\alpha_{n}\left(\mu F\left(T_{n} x_{n}\right)-\gamma Q x_{n}\right)-p\right] \\
& +\left(I-s_{n} V\right) p-\left(1-s_{n}\right) p \|^{2} \\
& =\|\left(I-s_{n} V\right)\left(T_{n} G v_{n}-p\right) \\
& +s_{n}\left[\alpha_{n}\left(\gamma Q x_{n}-\mu F p\right)+\left(I-\alpha_{n} \mu F\right) T_{n} x_{n}\right. \\
& \left.-\left(I-\alpha_{n} \mu F\right) T_{n} p\right]+s_{n}(I-V) p \|^{2} \\
& =\|\left(I-s_{n} V\right)\left(T_{n} G v_{n}-p\right) \\
& +s_{n}\left[\alpha_{n} \gamma\left(Q x_{n}-Q p\right)+\left(I-\alpha_{n} \mu F\right) T_{n} x_{n}\right. \\
& \left.-\left(I-\alpha_{n} \mu F\right) T_{n} p\right] \\
& +s_{n}\left[(I-V) p+\alpha_{n}(\gamma Q-\mu F) p\right] \|^{2} \\
& \leq \|\left(I-s_{n} V\right)\left(T_{n} G v_{n}-p\right) \\
& +s_{n}\left[\alpha_{n} \gamma\left(Q x_{n}-Q p\right)+\left(I-\alpha_{n} \mu F\right) T_{n} x_{n}\right. \\
& \left.-\left(I-\alpha_{n} \mu F\right) T_{n} p\right] \|^{2} \\
& +2 s_{n}\left\langle(I-V) p+\alpha_{n}(\gamma Q-\mu F) p, x_{n}-p\right\rangle \\
& \leq\left[\left\|\left(I-s_{n} V\right)\left(T_{n} G v_{n}-p\right)\right\|\right. \\
& +s_{n} \| \alpha_{n} \gamma\left(Q x_{n}-Q p\right)+\left(I-\alpha_{n} \mu F\right) T_{n} x_{n} \\
& \left.-\left(I-\alpha_{n} \mu F\right) T_{n} p \|\right]^{2} \\
& +2 s_{n}\left\langle(I-V) p+\alpha_{n}(\gamma Q-\mu F) p, x_{n}-p\right\rangle \\
& \leq\left[\left(1-s_{n} \bar{\gamma}\right)\left\|G v_{n}-p\right\|\right. \\
& \left.+s_{n}\left(\alpha_{n} \gamma l\left\|x_{n}-p\right\|+\left(1-\alpha_{n} \tau\right)\left\|x_{n}-p\right\|\right)\right]^{2} \\
& +2 s_{n}\left\langle(I-V) p+\alpha_{n}(\gamma Q-\mu F) p, x_{n}-p\right\rangle \\
& \leq\left[\left(1-s_{n} \bar{\gamma}\right)\left\|G v_{n}-p\right\|+s_{n}\left(1-\alpha_{n}(\tau-\gamma l)\right)\left\|x_{n}-p\right\|\right]^{2} \\
& +2 s_{n}\left\langle(I-V) p+\alpha_{n}(\gamma Q-\mu F) p, x_{n}-p\right\rangle \\
& =\left[\left(1-s_{n} \bar{\gamma}\right)\left\|G v_{n}-p\right\|+s_{n} \bar{\gamma} \frac{\left(1-\alpha_{n}(\tau-\gamma l)\right)}{\bar{\gamma}}\left\|x_{n}-p\right\|\right]^{2} \\
& +2 s_{n}\left\langle(I-V) p+\alpha_{n}(\gamma Q-\mu F) p, x_{n}-p\right\rangle \\
& \leq\left(1-s_{n} \bar{\gamma}\right)\left\|G v_{n}-p\right\|^{2}+s_{n} \bar{\gamma} \frac{\left(1-\alpha_{n}(\tau-\gamma l)\right)^{2}}{\bar{\gamma}^{2}}\left\|x_{n}-p\right\|^{2} \\
& +2 s_{n}\left\langle(I-V) p+\alpha_{n}(\gamma Q-\mu F) p, x_{n}-p\right\rangle \\
& \leq\left(1-s_{n} \bar{\gamma}\right)\left\|G v_{n}-p\right\|^{2}+s_{n}\left(1-\alpha_{n}(\tau-\gamma l)\right)\left\|x_{n}-p\right\|^{2}
\end{aligned}
$$

$$
\begin{aligned}
& +2 s_{n}\left\langle(I-V) p+\alpha_{n}(\gamma Q-\mu F) p, x_{n}-p\right\rangle \\
\leq & \left(1-s_{n} \bar{\gamma}\right)\left\|G v_{n}-p\right\|^{2}+s_{n}\left\|x_{n}-p\right\|^{2} \\
& +2 s_{n}\left\langle(I-V) p+\alpha_{n}(\gamma Q-\mu F) p, x_{n}-p\right\rangle \\
\leq & \left(1-s_{n} \bar{\gamma}\right)\left\|v_{n}-p\right\|^{2}+s_{n}\left\|x_{n}-p\right\|^{2} \\
& +2 s_{n}\left\langle(I-V) p+\alpha_{n}(\gamma Q-\mu F) p, x_{n}-p\right\rangle \\
\leq & \left(1-s_{n} \bar{\gamma}\right)\left\|u_{n}-p\right\|^{2}+s_{n}\left\|x_{n}-p\right\|^{2} \\
& +2 s_{n}\left\langle(I-V) p+\alpha_{n}(\gamma Q-\mu F) p, x_{n}-p\right\rangle \\
= & \left(1-s_{n}(\bar{\gamma}-1)\right)\left\|x_{n}-p\right\|^{2} \\
& +2 s_{n}(\|(I-V) p\|+\|(\gamma Q-\mu F) p\|)\left\|x_{n}-p\right\|,
\end{aligned}
$$

which implies that

$$
\left\|x_{n}-p\right\| \leq \frac{2}{\bar{\gamma}-1}(\|(I-V) p\|+\|(\gamma Q-\mu F) p\|) .
$$

Hence $\left\{x_{n}\right\}$ is bounded. So, according to (55) and (57) we know that $\left\{u_{n}\right\},\left\{v_{n}\right\},\left\{G v_{n}\right\},\left\{T_{n} G v_{n}\right\},\left\{T_{n} x_{n}\right\},\left\{Q x_{n}\right\}$, and $\left\{F T_{n} x_{n}\right\}$ are bounded.

Next let us show that $\left\|u_{n}-x_{n}\right\| \rightarrow 0,\left\|v_{n}-u_{n}\right\| \rightarrow 0$, $\left\|v_{n}-G v_{n}\right\| \rightarrow 0$ and $\left\|v_{n}-P_{C}(I-(2 / L) \nabla f) v_{n}\right\| \rightarrow 0$ as $n \rightarrow \infty$. Indeed, combining (55) and (59), we obtain

$$
\begin{aligned}
\| x_{n}- & p \|^{2} \\
\leq & \left(1-s_{n} \bar{\gamma}\right)\left\|u_{n}-p\right\|^{2}+s_{n}\left\|x_{n}-p\right\|^{2} \\
& +2 s_{n}\left\langle(I-V) p+\alpha_{n}(\gamma Q-\mu F) p, x_{n}-p\right\rangle \\
\leq & \left(1-s_{n} \bar{\gamma}\right)\left[\left\|x_{n}-p\right\|^{2}+r_{n}\left(r_{n}-2 \zeta\right)\left\|A x_{n}-A p\right\|^{2}\right] \\
& +s_{n}\left\|x_{n}-p\right\|^{2} \\
& +2 s_{n}\left\langle(I-V) p+\alpha_{n}(\gamma Q-\mu F) p, x_{n}-p\right\rangle \\
\leq & \left(1-s_{n}(\bar{\gamma}-1)\right)\left\|x_{n}-p\right\|^{2} \\
& +\left(1-s_{n} \bar{\gamma}\right) r_{n}\left(r_{n}-2 \zeta\right)\left\|A x_{n}-A p\right\|^{2} \\
& +2 s_{n}(\|(I-V) p\|+\|(\gamma Q-\mu F) p\|)\left\|x_{n}-p\right\| \\
\leq & \left\|x_{n}-p\right\|^{2} \\
& +\left(1-s_{n} \bar{\gamma}\right) r_{n}\left(r_{n}-2 \zeta\right)\left\|A x_{n}-A p\right\|^{2} \\
& +2 s_{n}(\|(I-V) p\|+\|(\gamma Q-\mu F) p\|)\left\|x_{n}-p\right\|,
\end{aligned}
$$

which immediately yields

$$
\begin{aligned}
& \left(1-s_{n} \bar{\gamma}\right) a(2 \zeta-\widehat{a})\left\|A x_{n}-A p\right\|^{2} \\
& \quad \leq\left(1-s_{n} \bar{\gamma}\right) r_{n}\left(2 \zeta-r_{n}\right)\left\|A x_{n}-A p\right\|^{2} \\
& \quad \leq 2 s_{n}(\|(I-V) p\|+\|(\gamma Q-\mu F) p\|)\left\|x_{n}-p\right\| .
\end{aligned}
$$


From $\lim _{n \rightarrow \infty} s_{n}=0$ and the boundedness of $\left\{x_{n}\right\}$ we conclude that

$$
\lim _{n \rightarrow \infty}\left\|A x_{n}-A p\right\|=0
$$

Furthermore, from the firm nonexpansivity of $S_{r_{n}}^{(\Theta, \varphi)}$, we have

$$
\begin{aligned}
& \left\|u_{n}-p\right\|^{2} \\
& =\left\|S_{r_{n}}^{(\Theta, \varphi)}\left(I-r_{n} A\right) x_{n}-S_{r_{n}}^{(\Theta, \varphi)}\left(I-r_{n} A\right) p\right\|^{2} \\
& \leq\left\langle\left(I-r_{n} A\right) x_{n}-\left(I-r_{n} A\right) p, u_{n}-p\right\rangle \\
& =\frac{1}{2}\left[\left\|\left(I-r_{n} A\right) x_{n}-\left(I-r_{n} A\right) p\right\|^{2}+\left\|u_{n}-p\right\|^{2}\right. \\
& \left.\quad-\left\|\left(I-r_{n} A\right) x_{n}-\left(I-r_{n} A\right) p-\left(u_{n}-p\right)\right\|^{2}\right] \\
& \leq \frac{1}{2}\left[\left\|x_{n}-p\right\|^{2}+\left\|u_{n}-p\right\|^{2}\right. \\
& \left.\quad-\left\|x_{n}-u_{n}-r_{n}\left(A x_{n}-A p\right)\right\|^{2}\right] \\
& =\frac{1}{2}\left[\left\|x_{n}-p\right\|^{2}+\left\|u_{n}-p\right\|^{2}-\left\|x_{n}-u_{n}\right\|^{2}\right. \\
& \left.\quad+2 r_{n}\left\langle A x_{n}-A p, x_{n}-u_{n}\right\rangle-r_{n}^{2}\left\|A x_{n}-A p\right\|^{2}\right],
\end{aligned}
$$

which leads to

$$
\begin{aligned}
\left\|u_{n}-p\right\|^{2} \leq & \left\|x_{n}-p\right\|^{2}-\left\|x_{n}-u_{n}\right\|^{2} \\
& +2 r_{n}\left\|A x_{n}-A p\right\|\left\|x_{n}-u_{n}\right\| .
\end{aligned}
$$

From (59) and (65), we get

$$
\begin{aligned}
\left\|x_{n}-p\right\|^{2} \leq & \left(1-s_{n} \bar{\gamma}\right)\left\|u_{n}-p\right\|^{2}+s_{n}\left\|x_{n}-p\right\|^{2} \\
& +2 s_{n}\left\langle(I-V) p+\alpha_{n}(\gamma Q-\mu F) p, x_{n}-p\right\rangle \\
\leq & \left(1-s_{n} \bar{\gamma}\right) \\
& \times\left[\left\|x_{n}-p\right\|^{2}-\left\|x_{n}-u_{n}\right\|^{2}\right. \\
& \left.+2 r_{n}\left\|A x_{n}-A p\right\|\left\|x_{n}-u_{n}\right\|\right] \\
& +s_{n}\left\|x_{n}-p\right\|^{2} \\
& +2 s_{n}(\|(I-V) p\|+\|(\gamma Q-\mu F) p\|)\left\|x_{n}-p\right\| \\
\leq & \left(1-s_{n}(\bar{\gamma}-1)\right)\left\|x_{n}-p\right\|^{2} \\
& -\left(1-s_{n} \bar{\gamma}\right)\left\|x_{n}-u_{n}\right\|^{2} \\
& +2 r_{n}\left\|A x_{n}-A p\right\|\left\|x_{n}-u_{n}\right\| \\
& +2 s_{n}(\|(I-V) p\|+\|(\gamma Q-\mu F) p\|)\left\|x_{n}-p\right\| \\
\leq & \left\|x_{n}-p\right\|^{2}-\left(1-s_{n} \bar{\gamma}\right)\left\|x_{n}-u_{n}\right\|^{2} \\
& +2 r_{n}\left\|A x_{n}-A p\right\|\left\|x_{n}-u_{n}\right\| \\
& +2 s_{n}(\|(I-V) p\|+\|(\gamma Q-\mu F) p\|)\left\|x_{n}-p\right\|,
\end{aligned}
$$

which hence implies that

$$
\begin{aligned}
(1- & \left.s_{n} \bar{\gamma}\right)\left\|x_{n}-u_{n}\right\|^{2} \\
\leq & 2 r_{n}\left\|A x_{n}-A p\right\|\left\|x_{n}-u_{n}\right\| \\
& +2 s_{n}(\|(I-V) p\|+\|(\gamma Q-\mu F) p\|)\left\|x_{n}-p\right\| \\
\leq & 2 \widehat{a}\left\|A x_{n}-A p\right\|\left\|x_{n}-u_{n}\right\| \\
& +2 s_{n}(\|(I-V) p\|+\|(\gamma Q-\mu F) p\|)\left\|x_{n}-p\right\| .
\end{aligned}
$$

Since $\lim _{n \rightarrow \infty} s_{n}=0$ and $\left\{x_{n}\right\}$ and $\left\{u_{n}\right\}$ are bounded sequences, it follows from (63) that

$$
\lim _{n \rightarrow \infty}\left\|x_{n}-u_{n}\right\|=0 \text {. }
$$

Next we show that $\lim _{n \rightarrow \infty}\left\|B_{i} \Lambda_{n}^{i} u_{n}-B_{i} p\right\|=0, i=$ $1,2, \ldots, N$. As a matter of fact, combining (55), (56), and (59), we have

$$
\begin{aligned}
\| x_{n}- & p \|^{2} \\
\leq & \left(1-s_{n} \bar{\gamma}\right)\left\|v_{n}-p\right\|^{2}+s_{n}\left\|x_{n}-p\right\|^{2} \\
& +2 s_{n}\left\langle(I-V) p+\alpha_{n}(\gamma Q-\mu F) p, x_{n}-p\right\rangle \\
\leq & \left(1-s_{n} \bar{\gamma}\right)\left[\left\|u_{n}-p\right\|^{2}+\lambda_{i, n}\left(\lambda_{i, n}-2 \eta_{i}\right)\right. \\
& \left.\quad \times\left\|B_{i} \Lambda_{n}^{i-1} u_{n}-B_{i} p\right\|^{2}\right]+s_{n}\left\|x_{n}-p\right\|^{2} \\
& +2 s_{n}(\|(I-V) p\|+\|(\gamma Q-\mu F) p\|)\left\|x_{n}-p\right\| \\
\leq & \left(1-s_{n} \bar{\gamma}\right) \\
& \times\left[\left\|x_{n}-p\right\|^{2}+\lambda_{i, n}\left(\lambda_{i, n}-2 \eta_{i}\right)\left\|B_{i} \Lambda_{n}^{i-1} u_{n}-B_{i} p\right\|^{2}\right] \\
& +s_{n}\left\|x_{n}-p\right\|^{2} \\
& +2 s_{n}(\|(I-V) p\|+\|(\gamma Q-\mu F) p\|)\left\|x_{n}-p\right\| \\
= & \left(1-s_{n}(\bar{\gamma}-1)\right)\left\|x_{n}-p\right\|^{2} \\
& +\left(1-s_{n} \bar{\gamma}\right) \lambda_{i, n}\left(\lambda_{i, n}-2 \eta_{i}\right)\left\|B_{i} \Lambda_{n}^{i-1} u_{n}-B_{i} p\right\|^{2} \\
& +2 s_{n}(\|(I-V) p\|+\|(\gamma Q-\mu F) p\|)\left\|x_{n}-p\right\| \\
\leq & \left\|x_{n}-p\right\|^{2}+\left(1-s_{n} \bar{\gamma}\right) \lambda_{i, n}\left(\lambda_{i, n}-2 \eta_{i}\right) \\
& \times\left\|B_{i} \Lambda_{n}^{i-1} u_{n}-B_{i} p\right\|^{2} \\
& +2 s_{n}(\|(I-V) p\|+\|(\gamma Q-\mu F) p\|)\left\|x_{n}-p\right\|,
\end{aligned}
$$

which leads to

$$
\begin{aligned}
& \left(1-s_{n} \bar{\gamma}\right) \lambda_{i, n}\left(2 \eta_{i}-\lambda_{i, n}\right)\left\|B_{i} \Lambda_{n}^{i-1} u_{n}-B_{i} p\right\|^{2} \\
& \quad \leq 2 s_{n}(\|(I-V) p\|+\|(\gamma Q-\mu F) p\|)\left\|x_{n}-p\right\| .
\end{aligned}
$$


Since $\lim _{n \rightarrow \infty} s_{n}=0,\left\{\lambda_{i, n}\right\} \subset\left[a_{i}, b_{i}\right] \subset\left(0,2 \eta_{i}\right), i=$ $1,2, \ldots, N$, and $\left\{x_{n}\right\}$ is bounded, it follows that

$$
\lim _{n \rightarrow \infty}\left\|B_{i} \Lambda_{n}^{i-1} u_{n}-B_{i} p\right\|=0 .
$$

Also, by Lemma 15, we obtain that for each $i \in\{1,2, \ldots, N\}$

$$
\begin{aligned}
\left\|\Lambda_{n}^{i} u_{n}-p\right\|^{2} & \\
= & \left\|J_{R_{i}, \lambda_{i, n}}\left(I-\lambda_{i, n} B_{i}\right) \Lambda_{n}^{i-1} u_{n}-J_{R_{i}, \lambda_{i, n}}\left(I-\lambda_{i, n} B_{i}\right) p\right\|^{2} \\
\leq & \left\langle\left(I-\lambda_{i, n} B_{i}\right) \Lambda_{n}^{i-1} u_{n}-\left(I-\lambda_{i, n} B_{i}\right) p, \Lambda_{n}^{i} u_{n}-p\right\rangle \\
= & \frac{1}{2}\left(\left\|\left(I-\lambda_{i, n} B_{i}\right) \Lambda_{n}^{i-1} u_{n}-\left(I-\lambda_{i, n} B_{i}\right) p\right\|^{2}+\left\|\Lambda_{n}^{i} u_{n}-p\right\|^{2}\right. \\
& \left.\quad-\left\|\left(I-\lambda_{i, n} B_{i}\right) \Lambda_{n}^{i-1} u_{n}-\left(I-\lambda_{i, n} B_{i}\right) p-\left(\Lambda_{n}^{i} u_{n}-p\right)\right\|^{2}\right) \\
\leq & \frac{1}{2}\left(\left\|\Lambda_{n}^{i-1} u_{n}-p\right\|^{2}+\left\|\Lambda_{n}^{i} u_{n}-p\right\|^{2}\right. \\
\quad & \left.\quad\left\|\Lambda_{n}^{i-1} u_{n}-\Lambda_{n}^{i} u_{n}-\lambda_{i, n}\left(B_{i} \Lambda_{n}^{i-1} u_{n}-B_{i} p\right)\right\|^{2}\right) \\
\leq & \frac{1}{2}\left(\left\|u_{n}-p\right\|^{2}+\left\|\Lambda_{n}^{i} u_{n}-p\right\|^{2}\right. \\
\quad & \left.\quad\left\|\Lambda_{n}^{i-1} u_{n}-\Lambda_{n}^{i} u_{n}-\lambda_{i, n}\left(B_{i} \Lambda_{n}^{i-1} u_{n}-B_{i} p\right)\right\|^{2}\right)
\end{aligned}
$$

which yields

$$
\begin{aligned}
\left\|\Lambda_{n}^{i} u_{n}-p\right\|^{2} \leq & \left\|u_{n}-p\right\|^{2} \\
& -\left\|\Lambda_{n}^{i-1} u_{n}-\Lambda_{n}^{i} u_{n}-\lambda_{i, n}\left(B_{i} \Lambda_{n}^{i-1} u_{n}-B_{i} p\right)\right\|^{2} \\
= & \left\|u_{n}-p\right\|^{2}-\left\|\Lambda_{n}^{i-1} u_{n}-\Lambda_{n}^{i} u_{n}\right\|^{2} \\
& -\lambda_{i, n}^{2}\left\|B_{i} \Lambda_{n}^{i-1} u_{n}-B_{i} p\right\|^{2} \\
& +2 \lambda_{i, n}\left\langle\Lambda_{n}^{i-1} u_{n}-\Lambda_{n}^{i} u_{n}, B_{i} \Lambda_{n}^{i-1} u_{n}-B_{i} p\right\rangle \\
\leq & \left\|u_{n}-p\right\|^{2}-\left\|\Lambda_{n}^{i-1} u_{n}-\Lambda_{n}^{i} u_{n}\right\|^{2} \\
& +2 \lambda_{i, n}\left\|\Lambda_{n}^{i-1} u_{n}-\Lambda_{n}^{i} u_{n}\right\|\left\|B_{i} \Lambda_{n}^{i-1} u_{n}-B_{i} p\right\|
\end{aligned}
$$

Thus, utilizing Lemma 8, from (41), (55), (56), (59), and (73) we have

$$
\begin{aligned}
\| x_{n}- & p \|^{2} \\
\leq & \left(1-s_{n} \bar{\gamma}\right)\left\|v_{n}-p\right\|^{2}+s_{n}\left\|x_{n}-p\right\|^{2} \\
& +2 s_{n}\left\langle(I-V) p+\alpha_{n}(\gamma Q-\mu F) p, x_{n}-p\right\rangle \\
\leq & \left(1-s_{n} \bar{\gamma}\right)\left\|\Lambda_{n}^{i} u_{n}-p\right\|^{2}+s_{n}\left\|x_{n}-p\right\|^{2} \\
& +2 s_{n}\left\langle(I-V) p+\alpha_{n}(\gamma Q-\mu F) p, x_{n}-p\right\rangle
\end{aligned}
$$

$$
\begin{aligned}
\leq & \left(1-s_{n} \bar{\gamma}\right) \\
& \times\left[\left\|u_{n}-p\right\|^{2}-\left\|\Lambda_{n}^{i-1} u_{n}-\Lambda_{n}^{i} u_{n}\right\|^{2}\right. \\
& \left.+2 \lambda_{i, n}\left\|\Lambda_{n}^{i-1} u_{n}-\Lambda_{n}^{i} u_{n}\right\|\left\|B_{i} \Lambda_{n}^{i-1} u_{n}-B_{i} p\right\|\right] \\
& +s_{n}\left\|x_{n}-p\right\|^{2} \\
& +2 s_{n}\left(\|(I-V) p\|+\alpha_{n}\|(\gamma Q-\mu F) p\|\right)\left\|x_{n}-p\right\| \\
\leq & \left(1-s_{n} \bar{\gamma}\right)\left[\left\|x_{n}-p\right\|^{2}-\left\|\Lambda_{n}^{i-1} u_{n}-\Lambda_{n}^{i} u_{n}\right\|^{2}\right. \\
& \left.\quad+2 \lambda_{i, n}\left\|\Lambda_{n}^{i-1} u_{n}-\Lambda_{n}^{i} u_{n}\right\|\left\|B_{i} \Lambda_{n}^{i-1} u_{n}-B_{i} p\right\|\right] \\
& +s_{n}\left\|x_{n}-p\right\|^{2}+2 s_{n}\left(\|(I-V) p\|+\alpha_{n}\|(\gamma Q-\mu F) p\|\right) \\
& \times\left\|x_{n}-p\right\| \\
\leq & \left(1-s_{n}(\bar{\gamma}-1)\right) \\
& +
\end{aligned}
$$

which leads to

$$
\begin{aligned}
& \left(1-s_{n} \bar{\gamma}\right)\left\|\Lambda_{n}^{i-1} u_{n}-\Lambda_{n}^{i} u_{n}\right\|^{2} \\
& \quad \leq 2 \lambda_{i, n}\left\|\Lambda_{n}^{i-1} u_{n}-\Lambda_{n}^{i} u_{n}\right\|\left\|B_{i} \Lambda_{n}^{i-1} u_{n}-B_{i} p\right\| \\
& \quad+2 s_{n}\left(\|(I-V) p\|+\alpha_{n}\|(\gamma Q-\mu F) p\|\right)\left\|x_{n}-p\right\| .
\end{aligned}
$$

Since $\lim _{n \rightarrow \infty} s_{n}=0,\left\{\lambda_{i, n}\right\} \subset\left[a_{i}, b_{i}\right] \subset\left(0,2 \eta_{i}\right), i=$ $1,2, \ldots, N$ and $\left\{x_{n}\right\}$ and $\left\{u_{n}\right\}$ are bounded sequences, it follows from (71) that

$$
\lim _{n \rightarrow \infty}\left\|\Lambda_{n}^{i-1} u_{n}-\Lambda_{n}^{i} u_{n}\right\|=0 .
$$

From (76) we get

$$
\begin{aligned}
\left\|u_{n}-v_{n}\right\|= & \left\|\Lambda_{n}^{0} u_{n}-\Lambda_{n}^{N} u_{n}\right\| \\
\leq & \left\|\Lambda_{n}^{0} u_{n}-\Lambda_{n}^{1} u_{n}\right\|+\left\|\Lambda_{n}^{1} u_{n}-\Lambda_{n}^{2} u_{n}\right\| \\
& +\cdots+\left\|\Lambda_{n}^{N-1} u_{n}-\Lambda_{n}^{N} u_{n}\right\| \\
& \longrightarrow 0 \text { as } n \longrightarrow \infty .
\end{aligned}
$$

Taking into account that $\left\|x_{n}-v_{n}\right\| \leq\left\|x_{n}-u_{n}\right\|+\left\|u_{n}-v_{n}\right\|$, we conclude from (68) and (77) that

$$
\lim _{n \rightarrow \infty}\left\|x_{n}-v_{n}\right\|=0 \text {. }
$$


On the other hand, for simplicity, we write $\widetilde{p}=T_{v_{2}}^{\Theta_{2}}(I-$ $\left.v_{2} A_{2}\right) p, \widetilde{v}_{n}=T_{v_{2}}^{\Theta_{2}}\left(I-v_{2} A_{2}\right) v_{n}$ and $w_{n}=G v_{n}=T_{v_{1}}^{\Theta_{1}}(I-$ $\left.\nu_{1} A_{1}\right) \widetilde{v}_{n}$ for all $n \geq 1$. Then

$$
\begin{aligned}
p & =G p=T_{\nu_{1}}^{\Theta_{1}}\left(I-v_{1} A_{1}\right) \tilde{p} \\
& =T_{\nu_{1}}^{\Theta_{1}}\left(I-v_{1} A_{1}\right) T_{\nu_{2}}^{\Theta_{2}}\left(I-v_{2} A_{2}\right) p .
\end{aligned}
$$

We now show that $\lim _{n \rightarrow \infty}\left\|G v_{n}-v_{n}\right\|=0$, that is, $\lim _{n \rightarrow \infty}\left\|w_{n}-v_{n}\right\|=0$. As a matter of fact, for $p \in \Omega$, it follows from (56), (57), and (59) that

$$
\begin{aligned}
\| x_{n}- & p \|^{2} \\
\leq & \left(1-s_{n} \bar{\gamma}\right)\left\|G v_{n}-p\right\|^{2}+s_{n}\left\|x_{n}-p\right\|^{2} \\
& +2 s_{n}\left\langle(I-V) p+\alpha_{n}(\gamma Q-\mu F) p, x_{n}-p\right\rangle \\
= & \left(1-s_{n} \bar{\gamma}\right)\left\|w_{n}-p\right\|^{2}+s_{n}\left\|x_{n}-p\right\|^{2} \\
& +2 s_{n}\left\langle(I-V) p+\alpha_{n}(\gamma Q-\mu F) p, x_{n}-p\right\rangle \\
\leq & \left(1-s_{n} \bar{\gamma}\right)\left\|w_{n}-p\right\|^{2}+s_{n}\left\|x_{n}-p\right\|^{2} \\
& +2 s_{n}(\|(I-V) p\|+\|(\gamma Q-\mu F) p\|)\left\|x_{n}-p\right\| \\
\leq & \left(1-s_{n} \bar{\gamma}\right) \\
& \times\left[\left\|\widetilde{v}_{n}-\tilde{p}\right\|^{2}+v_{1}\left(v_{1}-2 \zeta_{1}\right)\left\|A_{1} \widetilde{v}_{n}-A_{1} \tilde{p}\right\|^{2}\right] \\
& +s_{n}\left\|x_{n}-p\right\|^{2}+2 s_{n}(\|(I-V) p\|+\|(\gamma Q-\mu F) p\|) \\
& \times\left\|x_{n}-p\right\| \\
\leq & \left(1-s_{n} \bar{\gamma}\right) \\
& \times\left[\left\|v_{n}-p\right\|^{2}+v_{2}\left(v_{2}-2 \zeta_{2}\right)\left\|A_{2} v_{n}-A_{2} p\right\|^{2}\right. \\
& \left.+v_{1}\left(v_{1}-2 \zeta_{1}\right)\left\|A_{1} \widetilde{v}_{n}-A_{1} \tilde{p}\right\|^{2}\right] \\
& +2 s_{n}(\|(I-V) p\|+\|(\gamma Q-\mu F) p\|)\left\|x_{n}-p\right\| \\
& +s_{n}\left\|x_{n}-p\right\|^{2}+2 s_{n}(\|(I-V) p\|+\|(\gamma Q-\mu F) p\|) \\
& \times\left\|x_{n}-p\right\| \\
\leq & \left(1-s_{n} \bar{\gamma}\right) \\
& \times\left[\left\|x_{n}-p\right\|^{2}+v_{2}\left(v_{2}-2 \zeta_{2}\right)\left\|A_{2} v_{n}-A_{2} p\right\|^{2}\right. \\
& +2 s_{n}(\|(I-V) p\|+\|(\gamma Q-\mu F) p\|)\left\|x_{n}-p\right\| \\
& \times\left[v_{2}\left(v_{2}-2 \zeta_{2}\right)\left\|A_{2} v_{n}-A_{2} p\right\|^{2}\right. \\
& \\
&
\end{aligned}
$$

$$
\begin{aligned}
& \leq\left\|x_{n}-p\right\|^{2}+\left(1-s_{n} \bar{\gamma}\right) \\
& \quad \times\left[v_{2}\left(\nu_{2}-2 \zeta_{2}\right)\left\|A_{2} v_{n}-A_{2} p\right\|^{2}\right. \\
& \left.\quad+v_{1}\left(\nu_{1}-2 \zeta_{1}\right)\left\|A_{1} \widetilde{v}_{n}-A_{1} \tilde{p}\right\|^{2}\right] \\
& \quad+2 s_{n}(\|(I-V) p\|+\|(\gamma Q-\mu F) p\|)\left\|x_{n}-p\right\|,
\end{aligned}
$$

which immediately implies that

$$
\begin{aligned}
&\left(1-s_{n} \bar{\gamma}\right)[ v_{2}\left(2 \zeta_{2}-v_{2}\right)\left\|A_{2} v_{n}-A_{2} p\right\|^{2} \\
&\left.+v_{1}\left(2 \zeta_{1}-v_{1}\right)\left\|A_{1} \widetilde{v}_{n}-A_{1} \widetilde{p}\right\|^{2}\right] \\
& \leq 2 s_{n}(\|(I-V) p\|+\|(\gamma Q-\mu F) p\|)\left\|x_{n}-p\right\|,
\end{aligned}
$$

Since $\lim _{n \rightarrow \infty} s_{n}=0, v_{k} \in\left(0,2 \zeta_{k}\right), k=1,2$ and $\left\{x_{n}\right\}$ is bounded, it follows that

$$
\lim _{n \rightarrow \infty}\left\|A_{2} v_{n}-A_{2} p\right\|=0, \quad \lim _{n \rightarrow \infty}\left\|A_{1} \widetilde{v}_{n}-A_{1} \widetilde{p}\right\|=0 .
$$

Also, in terms of the firm nonexpansivity of $T_{\nu_{k}}^{\Theta_{k}}$ and the $\zeta_{k}$ inverse strong monotonicity of $A_{k}$ for $k=1$, 2, we obtain from $v_{k} \in\left(0,2 \zeta_{k}\right), k=1,2$, and (58) that

$$
\begin{aligned}
& \left\|\widetilde{v}_{n}-\tilde{p}\right\|^{2} \\
& =\left\|T_{v_{2}}^{\Theta_{2}}\left(I-v_{2} A_{2}\right) v_{n}-T_{v_{2}}^{\Theta_{2}}\left(I-v_{2} A_{2}\right) p\right\|^{2} \\
& \leq\left\langle\left(I-v_{2} A_{2}\right) v_{n}-\left(I-v_{2} A_{2}\right) p, \widetilde{v}_{n}-\tilde{p}\right\rangle \\
& =\frac{1}{2}\left[\left\|\left(I-v_{2} A_{2}\right) v_{n}-\left(I-v_{2} A_{2}\right) p\right\|^{2}+\left\|\widetilde{v}_{n}-\tilde{p}\right\|^{2}\right. \\
& \left.\quad-\left\|\left(I-v_{2} A_{2}\right) v_{n}-\left(I-v_{2} A_{2}\right) p-\left(\widetilde{v}_{n}-\tilde{p}\right)\right\|^{2}\right] \\
& \leq \frac{1}{2}\left[\left\|v_{n}-p\right\|^{2}+\left\|\widetilde{v}_{n}-\tilde{p}\right\|^{2}\right. \\
& \left.\quad-\left\|\left(v_{n}-\widetilde{v}_{n}\right)-v_{2}\left(A_{2} v_{n}-A_{2} p\right)-(p-\tilde{p})\right\|^{2}\right] \\
& =\frac{1}{2}\left[\left\|v_{n}-p\right\|^{2}+\left\|\widetilde{v}_{n}-\tilde{p}\right\|^{2}-\left\|\left(v_{n}-\widetilde{v}_{n}\right)-(p-\widetilde{p})\right\|^{2}\right. \\
& \quad+2 v_{2}\left\langle\left(v_{n}-\widetilde{v}_{n}\right)-(p-\tilde{p}), A_{2} v_{n}-A_{2} p\right\rangle \\
& \left.\quad-v_{2}^{2}\left\|A_{2} v_{n}-A_{2} p\right\|^{2}\right], \\
& \left\|w_{n}-p\right\|^{2} \\
& =\left\|T_{v_{1}}^{\Theta_{1}}\left(I-v_{1} A_{1}\right) \widetilde{v}_{n}-T_{v_{1}}^{\Theta_{1}}\left(I-v_{1} A_{1}\right) \tilde{p}\right\|^{2} \\
& \leq\left\langle\left(I-v_{1} A_{1}\right) \widetilde{v}_{n}-\left(I-v_{1} A_{1}\right) \tilde{p}, w_{n}-p\right\rangle \\
& =\frac{1}{2}\left[\left\|\left(I-v_{1} A_{1}\right) \widetilde{v}_{n}-\left(I-v_{1} A_{1}\right) \tilde{p}\right\|^{2}+\left\|w_{n}-p\right\|^{2}\right. \\
& \left.\quad-\left\|\left(I-v_{1} A_{1}\right) \widetilde{v}_{n}-\left(I-v_{1} A_{1}\right) \tilde{p}-\left(w_{n}-p\right)\right\|^{2}\right]
\end{aligned}
$$




$$
\begin{aligned}
\leq \frac{1}{2}[ & \left\|\widetilde{v}_{n}-\widetilde{p}\right\|^{2}+\left\|w_{n}-p\right\|^{2} \\
& -\left\|\left(\widetilde{v}_{n}-w_{n}\right)+(p-\widetilde{p})\right\|^{2} \\
& +2 v_{1}\left\langle A_{1} \widetilde{v}_{n}-A_{1} \widetilde{p},\left(\widetilde{v}_{n}-w_{n}\right)+(p-\widetilde{p})\right\rangle \\
& \left.-v_{1}^{2}\left\|A_{1} \widetilde{v}_{n}-A_{1} \tilde{p}\right\|^{2}\right] \\
\leq \frac{1}{2}[ & {\left[\left\|v_{n}-p\right\|^{2}+\left\|w_{n}-p\right\|^{2}\right.} \\
& -\left\|\left(\widetilde{v}_{n}-w_{n}\right)+(p-\widetilde{p})\right\|^{2} \\
& \left.+2 v_{1}\left\langle A_{1} \widetilde{v}_{n}-A_{1} \widetilde{p},\left(\widetilde{v}_{n}-w_{n}\right)+(p-\widetilde{p})\right\rangle\right] .
\end{aligned}
$$

Thus, we have

$$
\begin{aligned}
\left\|\widetilde{v}_{n}-\tilde{p}\right\|^{2} \leq & \left\|v_{n}-p\right\|^{2}-\left\|\left(v_{n}-\widetilde{v}_{n}\right)-(p-\tilde{p})\right\|^{2} \\
& +2 v_{2}\left\langle\left(v_{n}-\widetilde{v}_{n}\right)-(p-\tilde{p}), A_{2} v_{n}-A_{2} p\right\rangle \\
& -v_{2}^{2}\left\|A_{2} v_{n}-A_{2} p\right\|^{2}, \\
\left\|w_{n}-p\right\|^{2} \leq & \left\|v_{n}-p\right\|^{2}-\left\|\left(\widetilde{v}_{n}-w_{n}\right)+(p-\tilde{p})\right\|^{2} \\
& +2 v_{1}\left\|A_{1} \widetilde{v}_{n}-A_{1} \tilde{p}\right\|\left\|\left(\widetilde{v}_{n}-w_{n}\right)+(p-\tilde{p})\right\| .
\end{aligned}
$$

Consequently, from (57), (80), and (84) it follows that

$$
\begin{aligned}
\left\|x_{n}-p\right\|^{2} \leq & \left(1-s_{n} \bar{\gamma}\right) \\
& \times\left[\left\|\widetilde{v}_{n}-\tilde{p}\right\|^{2}+v_{1}\left(v_{1}-2 \zeta_{1}\right)\left\|A_{1} \widetilde{v}_{n}-A_{1} \widetilde{p}\right\|^{2}\right] \\
& +s_{n}\left\|x_{n}-p\right\|^{2} \\
& +2 s_{n}(\|(I-V) p\|+\|(\gamma Q-\mu F) p\|)\left\|x_{n}-p\right\| \\
\leq & \left(1-s_{n} \bar{\gamma}\right) \\
& \times\left\|\widetilde{v}_{n}-\tilde{p}\right\|^{2}+s_{n}\left\|x_{n}-p\right\|^{2} \\
& +2 s_{n}(\|(I-V) p\|+\|(\gamma Q-\mu F) p\|)\left\|x_{n}-p\right\| \\
\leq & \left(1-s_{n} \bar{\gamma}\right) \\
& \times\left[\left\|v_{n}-p\right\|^{2}-\left\|\left(v_{n}-\widetilde{v}_{n}\right)-(p-\widetilde{p})\right\|^{2}\right. \\
& +2 v_{2}\left\langle\left(v_{n}-\widetilde{v}_{n}\right)-(p-\widetilde{p}), A_{2} v_{n}-A_{2} p\right\rangle \\
& \left.-v_{2}^{2}\left\|A_{2} v_{n}-A_{2} p\right\|^{2}\right]+s_{n}\left\|x_{n}-p\right\|^{2} \\
& +2 s_{n}(\|(I-V) p\|+\|(\gamma Q-\mu F) p\|)\left\|x_{n}-p\right\| \\
\leq & \left(1-s_{n} \bar{\gamma}\right) \\
\times & \times\left[\left\|x_{n}-p\right\|^{2}-\left\|\left(v_{n}-\widetilde{v}_{n}\right)-(p-\tilde{p})\right\|^{2}\right. \\
& \left.+2 v_{2}\left\|\left(v_{n}-\widetilde{v}_{n}\right)-(p-\widetilde{p})\right\|\left\|A_{2} v_{n}-A_{2} p\right\|\right] \\
& +s_{n}\left\|x_{n}-p\right\|^{2}
\end{aligned}
$$

$$
\begin{aligned}
& +2 s_{n}(\|(I-V) p\|+\|(\gamma Q-\mu F) p\|)\left\|x_{n}-p\right\| \\
\leq & \left(1-s_{n}(\bar{\gamma}-1)\right) \\
& \times\left\|x_{n}-p\right\|^{2}-\left(1-s_{n} \bar{\gamma}\right)\left\|\left(v_{n}-\widetilde{v}_{n}\right)-(p-\tilde{p})\right\|^{2} \\
& +2 v_{2}\left\|\left(v_{n}-\widetilde{v}_{n}\right)-(p-\widetilde{p})\right\|\left\|A_{2} v_{n}-A_{2} p\right\| \\
& +2 s_{n}(\|(I-V) p\|+\|(\gamma Q-\mu F) p\|)\left\|x_{n}-p\right\| \\
\leq & \left\|x_{n}-p\right\|^{2}-\left(1-s_{n} \bar{\gamma}\right)\left\|\left(v_{n}-\widetilde{v}_{n}\right)-(p-\widetilde{p})\right\|^{2} \\
& +2 v_{2}\left\|\left(v_{n}-\widetilde{v}_{n}\right)-(p-\widetilde{p})\right\|\left\|A_{2} v_{n}-A_{2} p\right\| \\
& +2 s_{n}(\|(I-V) p\|+\|(\gamma Q-\mu F) p\|)\left\|x_{n}-p\right\|,
\end{aligned}
$$

which hence leads to

$$
\begin{aligned}
& \left(1-s_{n} \bar{\gamma}\right)\left\|\left(v_{n}-\widetilde{v}_{n}\right)-(p-\tilde{p})\right\|^{2} \\
& \leq 2 v_{2}\left\|\left(v_{n}-\widetilde{v}_{n}\right)-(p-\tilde{p})\right\|\left\|A_{2} v_{n}-A_{2} p\right\| \\
& \quad+2 s_{n}(\|(I-V) p\|+\|(\gamma Q-\mu F) p\|)\left\|x_{n}-p\right\| .
\end{aligned}
$$

Since $\lim _{n \rightarrow \infty} s_{n}=0$ and $\left\{x_{n}\right\},\left\{v_{n}\right\}$, and $\left\{\widetilde{v}_{n}\right\}$ are bounded sequences, we conclude from (82) that

$$
\lim _{n \rightarrow \infty}\left\|\left(v_{n}-\widetilde{v}_{n}\right)-(p-\widetilde{p})\right\|=0 .
$$

Consequently, from (57), (80), and (85) it follows that

$$
\begin{aligned}
\| x_{n}- & p \|^{2} \\
\leq & \left(1-s_{n} \bar{\gamma}\right)\left\|w_{n}-p\right\|^{2}+s_{n}\left\|x_{n}-p\right\|^{2} \\
& +2 s_{n}(\|(I-V) p\|+\|(\gamma Q-\mu F) p\|)\left\|x_{n}-p\right\| \\
\leq & \left(1-s_{n} \bar{\gamma}\right)\left[\left\|v_{n}-p\right\|^{2}-\left\|\left(\widetilde{v}_{n}-w_{n}\right)+(p-\widetilde{p})\right\|^{2}\right. \\
& \left.\quad+2 v_{1}\left\|A_{1} \widetilde{v}_{n}-A_{1} \tilde{p}\right\|\left\|\left(\widetilde{v}_{n}-w_{n}\right)+(p-\widetilde{p})\right\|\right] \\
& +s_{n}\left\|x_{n}-p\right\|^{2}+2 s_{n}(\|(I-V) p\|+\|(\gamma Q-\mu F) p\|) \\
& \times\left\|x_{n}-p\right\| \\
\leq & \left(1-s_{n} \bar{\gamma}\right) \\
& \times \\
& {\left[\left\|x_{n}-p\right\|^{2}-\left\|\left(\widetilde{v}_{n}-w_{n}\right)+(p-\tilde{p})\right\|^{2}\right.} \\
& \left.+2 v_{1}\left\|A_{1} \widetilde{v}_{n}-A_{1} \tilde{p}\right\|\left\|\left(\widetilde{v}_{n}-w_{n}\right)+(p-\widetilde{p})\right\|\right] \\
& +s_{n}\left\|x_{n}-p\right\|^{2}+2 s_{n}(\|(I-V) p\|+\|(\gamma Q-\mu F) p\|) \\
& \times\left\|x_{n}-p\right\| \\
\leq & \left(1-s_{n}(\bar{\gamma}-1)\right) \\
& \times\left\|x_{n}-p\right\|^{2}-\left(1-s_{n} \bar{\gamma}\right)\left\|\left(\widetilde{v}_{n}-w_{n}\right)+(p-\widetilde{p})\right\|^{2} \\
& +2 v_{1}\left\|A_{1} \widetilde{v}_{n}-A_{1} \tilde{p}\right\|\left\|\left(\widetilde{v}_{n}-w_{n}\right)+(p-\widetilde{p})\right\| \\
+ & 2 s_{n}(\|(I-V) p\|+\|(\gamma Q-\mu F) p\|)\left\|x_{n}-p\right\|
\end{aligned}
$$




$$
\begin{aligned}
\leq & \left\|x_{n}-p\right\|^{2}-\left(1-s_{n} \bar{\gamma}\right)\left\|\left(\widetilde{v}_{n}-w_{n}\right)+(p-\widetilde{p})\right\|^{2} \\
& +2 v_{1}\left\|A_{1} \widetilde{v}_{n}-A_{1} \tilde{p}\right\|\left\|\left(\widetilde{v}_{n}-w_{n}\right)+(p-\tilde{p})\right\| \\
& +2 s_{n}(\|(I-V) p\|+\|(\gamma Q-\mu F) p\|)\left\|x_{n}-p\right\|,
\end{aligned}
$$

which hence yields

$$
\begin{aligned}
& \left(1-s_{n} \bar{\gamma}\right)\left\|\left(\widetilde{v}_{n}-w_{n}\right)+(p-\tilde{p})\right\|^{2} \\
& \leq 2 v_{1}\left\|A_{1} \widetilde{v}_{n}-A_{1} \tilde{p}\right\|\left\|\left(\widetilde{v}_{n}-w_{n}\right)+(p-\tilde{p})\right\| \\
& \quad+2 s_{n}(\|(I-V) p\|+\|(\gamma Q-\mu F) p\|)\left\|x_{n}-p\right\| .
\end{aligned}
$$

Since $\lim _{n \rightarrow \infty} s_{n}=0$ and $\left\{x_{n}\right\},\left\{w_{n}\right\}$, and $\left\{\widetilde{v}_{n}\right\}$ are bounded sequences, we conclude from (82) that

$$
\lim _{n \rightarrow \infty}\left\|\left(\widetilde{v}_{n}-w_{n}\right)+(p-\tilde{p})\right\|=0
$$

Note that

$$
\left\|v_{n}-w_{n}\right\| \leq\left\|\left(v_{n}-\widetilde{v}_{n}\right)-(p-\widetilde{p})\right\|+\left\|\left(\widetilde{v}_{n}-w_{n}\right)+(p-\widetilde{p})\right\| .
$$

Hence from (88) and (91) we get

$$
\lim _{n \rightarrow \infty}\left\|v_{n}-G v_{n}\right\|=\lim _{n \rightarrow \infty}\left\|v_{n}-w_{n}\right\|=0 .
$$

Also, it is clear from (41) that

$$
\begin{aligned}
x_{n} & -T_{n} G v_{n} \\
& =s_{n}\left[T_{n} x_{n}-\alpha_{n}\left(\mu F\left(T_{n} x_{n}\right)-\gamma Q x_{n}\right)-V T_{n} G v_{n}\right] .
\end{aligned}
$$

So, it follows from $\lim _{n \rightarrow \infty} s_{n}=0$ that

$$
\lim _{n \rightarrow \infty}\left\|x_{n}-T_{n} G v_{n}\right\|=0 .
$$

Observe that

$$
\begin{aligned}
& \left\|v_{n}-T_{n} v_{n}\right\| \\
& \quad \leq\left\|v_{n}-x_{n}\right\|+\left\|x_{n}-T_{n} G v_{n}\right\|+\left\|T_{n} G v_{n}-T_{n} v_{n}\right\| \\
& \quad \leq\left\|v_{n}-x_{n}\right\|+\left\|x_{n}-T_{n} G v_{n}\right\|+\left\|G v_{n}-v_{n}\right\| .
\end{aligned}
$$

Hence, from (78), (93), and (95) we have

$$
\lim _{n \rightarrow \infty}\left\|v_{n}-T_{n} v_{n}\right\|=0 .
$$

It is easy to see that

$$
\begin{aligned}
& \left\|P_{C}\left(I-\lambda_{n} \nabla f\right) v_{n}-v_{n}\right\| \\
& \quad=\left\|s_{n} v_{n}+\left(1-s_{n}\right) T_{n} v_{n}-v_{n}\right\| \\
& \quad=\left(1-s_{n}\right)\left\|T_{n} v_{n}-v_{n}\right\| \\
& \quad \leq\left\|T_{n} v_{n}-v_{n}\right\|,
\end{aligned}
$$

where $s_{n}=\left(\left(2-\lambda_{n} L\right) / 4\right) \in(0,(1 / 2))$ for each $\lambda_{n} \in(0,(2 / L))$. Hence we have

$$
\begin{aligned}
\| P_{C} & \left(I-\frac{2}{L} \nabla f\right) v_{n}-v_{n} \| \\
\leq & \left\|P_{C}\left(I-\frac{2}{L} \nabla f\right) v_{n}-P_{C}\left(I-\lambda_{n} \nabla f\right) v_{n}\right\| \\
& +\left\|P_{C}\left(I-\lambda_{n} \nabla f\right) v_{n}-v_{n}\right\| \\
\leq & \left\|\left(I-\frac{2}{L} \nabla f\right) v_{n}-\left(I-\lambda_{n} \nabla f\right) v_{n}\right\| \\
& +\left\|P_{C}\left(I-\lambda_{n} \nabla f\right) v_{n}-v_{n}\right\| \\
\leq & \left(\frac{2}{L}-\lambda_{n}\right)\left\|\nabla f\left(v_{n}\right)\right\|+\left\|T_{n} v_{n}-v_{n}\right\| .
\end{aligned}
$$

From the boundedness of $\left\{v_{n}\right\}, s_{n} \rightarrow 0\left(\Leftrightarrow \lambda_{n} \rightarrow(2 / L)\right)$ and $\left\|T_{n} v_{n}-v_{n}\right\| \rightarrow 0$ (due to (97)), it follows that

$$
\lim _{n \rightarrow \infty}\left\|v_{n}-P_{C}\left(I-\frac{2}{L} \nabla f\right) v_{n}\right\|=0
$$

Furthermore, we show that $\omega_{w}\left(x_{n}\right) \subset \Omega$. Indeed, since $\left\{x_{n}\right\}$ is bounded, there exists a subsequence $\left\{x_{n_{i}}\right\}$ of $\left\{x_{n}\right\}$ which converges weakly to some $w$. Note that $\lim _{n \rightarrow \infty} \| x_{n}-$ $u_{n} \|=0$ (due to (68)). Hence $u_{n_{i}} \rightarrow w$. Since $C$ is closed and convex, $C$ is weakly closed. So, we have $w \in C$. From (68), (76), and (78) we have that $u_{n_{i}} \rightarrow w, v_{n_{i}} \rightarrow w$ and $\Lambda_{n_{i}}^{m} u_{n_{i}} \rightarrow w$, where $m \in\{1,2, \ldots, N\}$. First, we prove that $w \in \cap_{m=1}^{N} I\left(B_{m}, R_{m}\right)$. However, the argument to show that $w$ lies in $I\left(B_{m}, R_{m}\right)$ is quite standard by using maximality and hence is omitted. Next, we show that $w \in \operatorname{GMEP}(\Theta, \varphi, A)$. In fact, from $u_{n}=S_{r_{n}}^{(\Theta, \varphi)}\left(I-r_{n} A\right) x_{n}$, we know that

$$
\begin{aligned}
& \Theta\left(u_{n}, y\right)+\varphi(y)-\varphi\left(u_{n}\right)+\left\langle A x_{n}, y-u_{n}\right\rangle \\
& +\frac{1}{r_{n}}\left\langle K^{\prime}\left(u_{n}\right)-K^{\prime}\left(x_{n}\right), y-u_{n}\right\rangle \geq 0, \quad \forall y \in C .
\end{aligned}
$$

From (H2) it follows that

$$
\begin{aligned}
\varphi(y)-\varphi\left(u_{n}\right)+\left\langle A x_{n}, y-u_{n}\right\rangle+\frac{1}{r_{n}} & \\
\times\left\langle K^{\prime}\left(u_{n}\right)-K^{\prime}\left(x_{n}\right), y-u_{n}\right\rangle \geq \Theta & \left(y, u_{n}\right), \\
& \forall y \in C .
\end{aligned}
$$

Replacing $n$ by $n_{i}$, we have

$$
\begin{aligned}
& \varphi(y)-\varphi\left(u_{n_{i}}\right)+\left\langle A x_{n_{i}}, y-u_{n_{i}}\right\rangle \\
& +\left\langle\frac{K^{\prime}\left(u_{n_{i}}\right)-K^{\prime}\left(x_{n_{i}}\right)}{r_{n_{i}}}, y-u_{n_{i}}\right\rangle \geq \Theta\left(y, u_{n_{i}}\right),
\end{aligned}
$$


Put $u_{t}=t y+(1-t) w$ for all $t \in(0,1]$ and $y \in C$. Then, from (103) we have

$$
\begin{aligned}
\left\langle u_{t}-\right. & \left.u_{n_{i}}, A u_{t}\right\rangle \\
\geq & \left\langle u_{t}-u_{n_{i}}, A u_{t}\right\rangle-\varphi\left(u_{t}\right)+\varphi\left(u_{n_{i}}\right) \\
& -\left\langle u_{t}-u_{n_{i}}, A x_{n_{i}}\right\rangle-\left\langle\frac{K^{\prime}\left(u_{n_{i}}\right)-K^{\prime}\left(x_{n_{i}}\right)}{r_{n_{i}}}, u_{t}-u_{n_{i}}\right\rangle \\
& +\Theta\left(u_{t}, u_{n_{i}}\right) \\
\geq & \left\langle u_{t}-u_{n_{i}}, A u_{t}-A u_{n_{i}}\right\rangle \\
& +\left\langle u_{t}-u_{n_{i}}, A u_{n_{i}}-A x_{n_{i}}\right\rangle-\varphi\left(u_{t}\right)+\varphi\left(u_{n_{i}}\right) \\
& -\left\langle\frac{K^{\prime}\left(u_{n_{i}}\right)-K^{\prime}\left(x_{n_{i}}\right)}{r_{n_{i}}}, u_{t}-u_{n_{i}}\right\rangle+\Theta\left(u_{t}, u_{n_{i}}\right) .
\end{aligned}
$$

Since $\left\|u_{n_{i}}-x_{n_{i}}\right\| \rightarrow 0$ as $i \rightarrow \infty$, we deduce from the Lipschitz continuity of $A$ and $K^{\prime}$ that $\left\|A u_{n_{i}}-A x_{n_{i}}\right\| \rightarrow 0$ and $\left\|K^{\prime}\left(u_{n_{i}}\right)-K^{\prime}\left(x_{n_{i}}\right)\right\| \rightarrow 0$ as $i \rightarrow \infty$. Furthermore, from the monotonicity of $A$, we have $\left\langle u_{t}-u_{n_{i}}, A u_{t}-A u_{n_{i}}\right\rangle \geq 0$. So, from (H4), the weakly lower semicontinuity of $\varphi,\left(\left(K^{\prime}\left(u_{n_{i}}\right)-\right.\right.$ $\left.\left.K^{\prime}\left(x_{n_{i}}\right)\right) / r_{n_{i}}\right) \rightarrow 0$, and $u_{n_{i}} \rightarrow w$, we have

$$
\left\langle u_{t}-w, A u_{t}\right\rangle \geq-\varphi\left(u_{t}\right)+\varphi(w)+\Theta\left(u_{t}, w\right), \quad \text { as } i \longrightarrow \infty \text {. }
$$

From (H1), (H4), and (105) we also have

$$
\begin{aligned}
0= & \Theta\left(u_{t}, u_{t}\right)+\varphi\left(u_{t}\right)-\varphi\left(u_{t}\right) \\
\leq & t \Theta\left(u_{t}, y\right)+(1-t) \Theta\left(u_{t}, w\right)+t \varphi(y) \\
& +(1-t) \varphi(w)-\varphi\left(u_{t}\right) \\
= & t\left[\Theta\left(u_{t}, y\right)+\varphi(y)-\varphi\left(u_{t}\right)\right] \\
& \quad+(1-t)\left[\Theta\left(u_{t}, w\right)+\varphi(w)-\varphi(w)-\varphi\left(u_{t}\right)\right] \\
\leq & t\left[\Theta\left(u_{t}, y\right)+\varphi(y)-\varphi\left(u_{t}\right)\right] \\
& +(1-t)\left\langle u_{t}-w, A u_{t}\right\rangle \\
= & t\left[\Theta\left(u_{t}, y\right)+\varphi(y)-\varphi\left(u_{t}\right)\right]+(1-t) t\left\langle y-w, A u_{t}\right\rangle,
\end{aligned}
$$

and hence

$$
0 \leq \Theta\left(u_{t}, y\right)+\varphi(y)-\varphi\left(u_{t}\right)+(1-t)\left\langle y-w, A u_{t}\right\rangle .
$$

Letting $t \rightarrow 0$, we have, for each $y \in C$,

$$
0 \leq \Theta(w, y)+\varphi(y)-\varphi(w)+\langle A w, y-w\rangle
$$

This implies that $w \in \operatorname{GMEP}(\Theta, \varphi, A)$. In addition, let us show that $w \in \operatorname{SGEP}(G) \cap \Gamma$. As a matter of fact, from (93), (100), $v_{n_{i}} \rightarrow w$, and Lemma 10, we deduce that $w \in \operatorname{SGEP}(G)$ and $w \in \operatorname{Fix}\left(P_{C}(I-(2 / L) \nabla f)\right)=\operatorname{VI}(C, \nabla f)=\Gamma$. Hence we get $w \epsilon$ $\operatorname{SGEP}(G) \cap \Gamma$. Therefore, $w \in \operatorname{GMEP}(\Theta, \varphi, A) \cap \operatorname{SGEP}(G) \cap$ $\cap_{i=1}^{N} I\left(B_{i}, R_{i}\right) \cap \Gamma=: \Omega$. This shows that $\omega_{w}\left(x_{n}\right) \subset \Omega$.

Finally, we prove that $\left\{x_{n}\right\}$ converges strongly as $\lambda_{n} \rightarrow$ $(2 / L)\left(\Leftrightarrow s_{n} \rightarrow 0\right)$ to $q \in \Omega$, which is the unique solution in $\Omega$ to the VIP (43). In fact, we note that, for $w \in \Omega$ with $x_{n_{i}} \rightarrow w$,

$$
\begin{aligned}
x_{n}-w= & \left(I-s_{n} V\right)\left(T_{n} G v_{n}-w\right) \\
+ & s_{n}\left[\alpha_{n} \gamma\left(Q x_{n}-Q w\right)+\left(I-\alpha_{n} \mu F\right) T_{n} x_{n}\right. \\
& \left.\quad-\left(I-\alpha_{n} \mu F\right) T_{n} w\right] \\
+ & s_{n}\left[(I-V) w+\alpha_{n}(\gamma Q-\mu F) w\right] .
\end{aligned}
$$

By (48), (57), and Lemma 14, we obtain that

$$
\begin{aligned}
& \left\|x_{n}-w\right\|^{2} \\
& =\left\langle\left(I-s_{n} V\right)\left(T_{n} G v_{n}-w\right), x_{n}-w\right\rangle \\
& +s_{n}\left[\alpha_{n} \gamma\left\langle\left(Q x_{n}-Q w\right), x_{n}-w\right\rangle\right. \\
& \left.+\left\langle\left(I-\alpha_{n} \mu F\right) T_{n} x_{n}-\left(I-\alpha_{n} \mu F\right) T_{n} w, x_{n}-w\right\rangle\right] \\
& +s_{n}\left\langle(I-V) w+\alpha_{n}(\gamma Q-\mu F) w, x_{n}-w\right\rangle \\
& \leq\left(1-s_{n} \bar{\gamma}\right)\left\|T_{n} G v_{n}-w\right\|\left\|x_{n}-w\right\| \\
& +s_{n}\left[\alpha_{n} \gamma\left\|Q x_{n}-Q w\right\|\left\|x_{n}-w\right\|\right. \\
& \left.+\left(1-\alpha_{n} \tau\right)\left\|x_{n}-w\right\|^{2}\right] \\
& +s_{n}\left\langle(I-V) w+\alpha_{n}(\gamma Q-\mu F) w, x_{n}-w\right\rangle \\
& \leq\left(1-s_{n} \bar{\gamma}\right)\left\|v_{n}-w\right\|\left\|x_{n}-w\right\| \\
& +s_{n}\left[\alpha_{n} \gamma l\left\|x_{n}-w\right\|^{2}+\left(1-\alpha_{n} \tau\right)\left\|x_{n}-w\right\|^{2}\right] \\
& +s_{n}\left\langle(I-V) w+\alpha_{n}(\gamma Q-\mu F) w, x_{n}-w\right\rangle \\
& \leq\left(1-s_{n} \bar{\gamma}\right)\left\|x_{n}-w\right\|^{2} \\
& +s_{n}\left[\alpha_{n} \gamma l\left\|x_{n}-w\right\|^{2}+\left(1-\alpha_{n} \tau\right)\left\|x_{n}-w\right\|^{2}\right] \\
& +s_{n}\left\langle(I-V) w+\alpha_{n}(\gamma Q-\mu F) w, x_{n}-w\right\rangle \\
& =\left(1-s_{n} \bar{\gamma}\right)\left\|x_{n}-w\right\|^{2}+s_{n}\left(1-\alpha_{n}(\tau-\gamma l)\right)\left\|x_{n}-w\right\|^{2} \\
& +s_{n}\left\langle(I-V) w+\alpha_{n}(\gamma Q-\mu F) w, x_{n}-w\right\rangle \\
& \leq\left(1-s_{n} \bar{\gamma}\right)\left\|x_{n}-w\right\|^{2}+s_{n}\left\|x_{n}-w\right\|^{2} \\
& +s_{n}\left\langle(I-V) w+\alpha_{n}(\gamma Q-\mu F) w, x_{n}-w\right\rangle \\
& =\left(1-s_{n}(\bar{\gamma}-1)\right)\left\|x_{n}-w\right\|^{2} \\
& +s_{n}\left\langle(I-V) w+\alpha_{n}(\gamma Q-\mu F) w, x_{n}-w\right\rangle,
\end{aligned}
$$


which hence leads to

$$
\left\|x_{n}-w\right\|^{2} \leq \frac{\left\langle(I-V) w+\alpha_{n}(\gamma Q-\mu F) w, x_{n}-w\right\rangle}{\bar{\gamma}-1} .
$$

In particular, we have

$$
\left\|x_{n_{i}}-w\right\|^{2} \leq \frac{\left\langle(I-V) w+\alpha_{n_{i}}(\gamma Q-\mu F) w, x_{n_{i}}-w\right\rangle}{\bar{\gamma}-1} .
$$

Since $x_{n_{i}} \rightarrow w$ and $\lim _{n \rightarrow \infty} \alpha_{n}=0$, it follows from (112) that $x_{n_{i}} \rightarrow w$ as $i \rightarrow \infty$.

Now we show that $w$ solves the VIP (43). As a matter of fact, from (55) and (59) we obtain that, for any $p \in \Omega$,

$$
\begin{aligned}
\left\|x_{n}-p\right\|^{2} \leq & \left(1-s_{n} \bar{\gamma}\right)\left\|u_{n}-p\right\|^{2}+s_{n}\left\|x_{n}-p\right\|^{2} \\
& +2 s_{n}\left\langle(I-V) p+\alpha_{n}(\gamma Q-\mu F) p, x_{n}-p\right\rangle \\
\leq & \left(1-s_{n} \bar{\gamma}\right)\left\|x_{n}-p\right\|^{2}+s_{n}\left\|x_{n}-p\right\|^{2} \\
& +2 s_{n}\left\langle(I-V) p+\alpha_{n}(\gamma Q-\mu F) p, x_{n}-p\right\rangle \\
= & \left(1-s_{n}(\bar{\gamma}-1)\right)\left\|x_{n}-p\right\|^{2} \\
& +2 s_{n}\left\langle(I-V) p+\alpha_{n}(\gamma Q-\mu F) p, x_{n}-p\right\rangle \\
\leq & \left\|x_{n}-p\right\|^{2} \\
& +2 s_{n}\left\langle(I-V) p+\alpha_{n}(\gamma Q-\mu F) p, x_{n}-p\right\rangle,
\end{aligned}
$$

which immediately implies that

$$
\left\langle(V-I) p+\alpha_{n}(\mu F-\gamma Q) p, x_{n}-p\right\rangle \leq 0 .
$$

Since $\alpha_{n} \rightarrow 0$ and $x_{n_{i}} \rightarrow w$, we get

$$
\langle(V-I) p, w-p\rangle \leq 0, \quad \forall p \in \Omega .
$$

By Minty's lemma, $w$ is a solution in $\Omega$ to the VIP (43). In terms of the uniqueness of solutions of VIP (43), we deduce that $w=q$ and $x_{n_{i}} \rightarrow q$ as $n \rightarrow \infty$. So, every weak convergence subsequence of $\left\{x_{n}\right\}$ converges strongly to the unique solution $q$ of VIP (43). Therefore, $\left\{x_{n}\right\}$ converges strongly to the unique solution $q$ of VIP (43). This completes the proof.

Corollary 20. Let $C$ be a nonempty closed convex subset of a real Hilbert space $H$. Let $f: C \rightarrow \mathbf{R}$ be a convex functional with L-Lipschitz continuous gradient $\nabla f$. Let $\Theta, \Theta_{1}, \Theta_{2}$ be three bifunctions from $\mathrm{C} \times \mathrm{C}$ to $\mathbf{R}$ satisfying (H1)-(H4) and let $\varphi: C \rightarrow \mathbf{R}$ be a lower semicontinuous and convex functional. Let $R_{i}: C \rightarrow 2^{H}$ be a maximal monotone mapping and let $A: H \rightarrow H$ and $A_{k}, B_{i}: C \rightarrow H$ be $\zeta$ inverse strongly monotone, $\zeta_{k}$-inverse strongly monotone, and $\eta_{i}$-inverse-strongly monotone, respectively, for $k=1,2$ and $i=1,2$. Let $V$ be a $\bar{\gamma}$-strongly positive bounded linear operator with $\bar{\gamma}>1$. Let $F: H \rightarrow H$ be a $\kappa$-Lipschitzian and $\eta$ strongly monotone operator with positive constants $\kappa, \eta>0$. Let $\mathrm{Q}: H \rightarrow H$ be an l-Lipschitzian mapping with constant $l \geq 0$. Let $0<\mu<\left(2 \eta / \kappa^{2}\right)$ and $0 \leq \gamma l<\tau$, where $\tau=1-\sqrt{1-\mu\left(2 \eta-\mu \kappa^{2}\right)}$. Assume that $\Omega:=\operatorname{GMEP}(\Theta, \varphi, A) \cap$ $\operatorname{SGEP}(G) \cap I\left(B_{1}, R_{1}\right) \cap I\left(B_{2}, R_{2}\right) \cap \Gamma \neq \emptyset$ where $G$ is defined as in Proposition CY. Let $\left\{r_{n}\right\}$ be a sequence in $(0,2 \zeta]$ and $\left\{\alpha_{n}\right\}$ a sequence in $(0,1]$ such that $\lim _{n \rightarrow \infty} \alpha_{n}=0$. Let $\left\{x_{n}\right\}$ be the sequence generated by

$$
\begin{gathered}
u_{n}=S_{r_{n}}^{(\Theta, \varphi)}\left(I-r_{n} A\right) x_{n}, \\
v_{n}=J_{R_{2}, \lambda_{2, n}}\left(I-\lambda_{2, n} B_{2}\right) J_{R_{1}, \lambda_{1, n}}\left(I-\lambda_{1, n} B_{1}\right) u_{n}, \\
x_{n}=\left(I-s_{n} V\right) T_{n} G v_{n} \\
+s_{n}\left[T_{n} x_{n}-\alpha_{n}\left(\mu F\left(T_{n} x_{n}\right)-\gamma Q x_{n}\right)\right], \quad \forall n \geq 1,
\end{gathered}
$$

where $P_{C}\left(I-\lambda_{n} \nabla f\right)=s_{n} I+\left(1-s_{n}\right) T_{n}$ (here $T_{n}$ is nonexpansive, $s_{n}=\left(\left(2-\lambda_{n} L\right) / 4\right) \in(0,(1 / 2))$ for each $\left.\lambda_{n} \in(0,(2 / L))\right)$. Suppose that the following conditions hold.

(i) $K: H \rightarrow \mathbf{R}$ is strongly convex with constant $\sigma>0$ and its derivative $K^{\prime}$ is Lipschitz continuous with constant $v>0$ such that the function $x \mapsto\left\langle y-x, K^{\prime}(x)\right\rangle$ is weakly upper semicontinuous for each $y \in H$.

(ii) for each $x \in H$, there exist a bounded subset $D_{x} \subset C$ and $z_{x} \in C$ such that for any $y \notin D_{x}$,

$\Theta\left(y, z_{x}\right)+\varphi\left(z_{x}\right)-\varphi(y)+\frac{1}{r}\left\langle K^{\prime}(y)-K^{\prime}(x), z_{x}-y\right\rangle<0$.

(iii) $s_{n} \in(0,(1 / 2))$ for each $\lambda_{n} \in(0,(2 / L)), \lim _{n \rightarrow \infty} s_{n}=$ $0\left(\Leftrightarrow \lim _{n \rightarrow \infty} \lambda_{n}=(2 / L)\right)$.

(iv) $v_{k} \in\left(0,2 \zeta_{k}\right), k=1,2$ and $\left\{\lambda_{i, n}\right\} \subset\left[a_{i}, b_{i}\right] \subset$ $\left(0,2 \eta_{i}\right), i=1,2$.

(v) $0<\liminf _{n \rightarrow \infty} r_{n} \leq \lim \sup _{n \rightarrow \infty} r_{n}<2 \zeta$.

Assume that $S_{r}^{(\Theta, \varphi)}$ is firmly nonexpansive. Then $\left\{x_{n}\right\}$ converges strongly as $\lambda_{n} \rightarrow(2 / L)\left(\Leftrightarrow s_{n} \rightarrow 0\right)$ to a point $q \in \Omega$, which is a unique solution in $\Omega$ to the VIP:

$$
\langle(I-V) q, p-q\rangle \leq 0, \quad \forall p \in \Omega .
$$

Corollary 21. Let $C$ be a nonempty closed convex subset of a real Hilbert space $H$. Let $f: C \rightarrow \mathbf{R}$ be a convex functional with L-Lipschitz continuous gradient $\nabla f$. Let $\Theta, \Theta_{1}, \Theta_{2}$ be three bifunctions from $\mathrm{C} \times \mathrm{C}$ to $\mathbf{R}$ satisfying $(\mathrm{H1})-(\mathrm{H} 4)$ and $\varphi: C \rightarrow$ $\mathbf{R}$ a lower semicontinuous and convex functional. Let $R: C \rightarrow$ $2^{H}$ be a maximal monotone mapping and let $A: H \rightarrow H$ and $A_{k}, B: C \rightarrow H$ be $\zeta$-inverse strongly monotone, $\zeta_{k^{-}}$ inverse strongly monotone, and $\xi$-inverse-strongly monotone, respectively, for $k=1,2$. Let $V$ be a $\bar{\gamma}$-strongly positive bounded linear operator with $\bar{\gamma}>1$. Let $F: H \rightarrow H$ be $a \kappa-$ Lipschitzian and $\eta$-strongly monotone operator with positive constants $\kappa, \eta>0$. Let $Q: H \rightarrow H$ be an l-Lipschitzian mapping with constant $l \geq 0$. Let $0<\mu<\left(2 \eta / \kappa^{2}\right)$ and $0 \leq \gamma l<\tau$, where $\tau=1-\sqrt{1-\mu\left(2 \eta-\mu \kappa^{2}\right)}$. Assume that $\Omega:=\operatorname{GMEP}(\Theta, \varphi, A) \cap \operatorname{SGEP}(G) \cap I(B, R) \cap \Gamma \neq \emptyset$ where $G$ is 
defined as in Proposition CY. Let $\left\{r_{n}\right\}$ be a sequence in $(0,2 \zeta]$ and $\left\{\alpha_{n}\right\}$ be a sequence in $(0,1]$ such that $\lim _{n \rightarrow \infty} \alpha_{n}=0$. Let $\left\{x_{n}\right\}$ be the sequence generated by

$$
\begin{gathered}
u_{n}=S_{r_{n}}^{(\Theta, \varphi)}\left(I-r_{n} A\right) x_{n}, \\
v_{n}=J_{R, \rho_{n}}\left(I-\rho_{n} B\right) u_{n}, \\
x_{n}=\left(I-s_{n} V\right) T_{n} G v_{n}+s_{n}\left[T_{n} x_{n}-\alpha_{n}\left(\mu F\left(T_{n} x_{n}\right)-\gamma Q x_{n}\right)\right],
\end{gathered}
$$$$
\forall n \geq 1 \text {, }
$$

where $P_{C}\left(I-\lambda_{n} \nabla f\right)=s_{n} I+\left(1-s_{n}\right) T_{n}$ (here $T_{n}$ is nonexpansive, $s_{n}=\left(\left(2-\lambda_{n} L\right) / 4\right) \in(0,(1 / 2))$ for each $\left.\lambda_{n} \in(0,(2 / L))\right)$. Suppose that the following conditions hold

(i) $\mathrm{K}: H \rightarrow \mathbf{R}$ is strongly convex with constant $\sigma>0$ and its derivative $K^{\prime}$ is Lipschitz continuous with constant $v>0$ such that the function $x \mapsto\left\langle y-x, K^{\prime}(x)\right\rangle$ is weakly upper semicontinuous for each $y \in H$;

(ii) for each $x \in H$, there exist a bounded subset $D_{x} \subset C$ and $z_{x} \in C$ such that for any $y \notin D_{x}$,

$$
\begin{aligned}
& \Theta\left(y, z_{x}\right)+\varphi\left(z_{x}\right)-\varphi(y) \\
& +\frac{1}{r}\left\langle K^{\prime}(y)-K^{\prime}(x), z_{x}-y\right\rangle<0 ;
\end{aligned}
$$

(iii) $s_{n} \in(0,(1 / 2))$ for each $\lambda_{n} \in(0,(2 / L)), \lim _{n \rightarrow \infty} s_{n}=$ $0\left(\Leftrightarrow \lim _{n \rightarrow \infty} \lambda_{n}=(2 / L)\right)$;

(iv) $\nu_{k} \in\left(0,2 \zeta_{k}\right), k=1,2$ and $\left\{\rho_{n}\right\} \subset[a, b] \subset(0,2 \xi)$;

(v) $0<\liminf _{n \rightarrow \infty} r_{n} \leq \limsup _{n \rightarrow \infty} r_{n}<2 \zeta$.

Assume that $S_{r}^{(\Theta, \varphi)}$ is firmly nonexpansive. Then $\left\{x_{n}\right\}$ converges strongly as $\lambda_{n} \rightarrow(2 / L)\left(\Leftrightarrow s_{n} \rightarrow 0\right)$ to a point $q \in \Omega$, which is a unique solution in $\Omega$ to the VIP:

$$
\langle(I-V) q, p-q\rangle \leq 0, \quad \forall p \in \Omega .
$$

We next provide one example to illustrate Corollary 21.

Example 22. Let $H=\mathbf{R}^{2}$ with inner product $\langle\cdot, \cdot\rangle$ and norm $\|\cdot\|$ which are defined by

$$
\langle x, y\rangle=a c+b d, \quad\|x\|=\sqrt{a^{2}+b^{2}}
$$

for all $x, y \in \mathbf{R}^{2}$ with $x=(a, b)$ and $y=(c, d)$. Let $C=$ $\{(a, a): a \in \mathbf{R}\}$ and $K(x)=(1 / 2)\|x\|^{2}$, for all $x \in \mathbf{R}^{2}$. Clearly, $C$ is a nonempty closed convex subset of a real Hilbert space $H=\mathbf{R}^{2}$. Let $f: C \rightarrow \mathbf{R}$ be a convex functional with $L$ Lipschitz continuous gradient $\nabla f$; for instance, putting $S=$ $\left\{\begin{array}{ll}2 / 3 & 1 / 3 \\ 1 / 3 & 2 / 3\end{array}\right\}$ and $f(x)=(1 / 2)\langle S x, x\rangle$, we obtain that $\|S\|=1$ and $\nabla f=S$ with Lipschitz constant $L=4$. Put $\Theta=\Theta_{1}=\Theta_{2}=0$ and $\varphi=0$. Then $\Theta, \Theta_{1}, \Theta_{2}$ are three bifunctions from $C \times C$ to $\mathbf{R}$ satisfying (H1)-(H4) and let $\varphi: C \rightarrow \mathbf{R}$ be a lower semicontinuous and convex functional. Let $R: C \rightarrow 2^{H}$ be a maximal monotone mapping, for instance, putting

$$
R v= \begin{cases}S v+N_{C} v, & \text { if } v \in C, \\ \emptyset, & \text { if } v \notin C,\end{cases}
$$

where $N_{C} v=\{w \in H:\langle v-u, w\rangle \geq 0$, for all $u \in C\}$. It is known that $R$ is maximal monotone and $0 \in R v$ if and only if $v \in V I(C, S)$. Put $A=\left\{\begin{array}{ll}3 / 5 & 2 / 5 \\ 2 / 5 & 3 / 5\end{array}\right\}, B=0, A_{1}=I-S=$ $\left\{\begin{array}{cc}1 / 3 & -1 / 3 \\ -1 / 3 & 1 / 3\end{array}\right\}, A_{2}=I-A=\left\{\begin{array}{cc}2 / 5 & -2 / 5 \\ -2 / 5 & 2 / 5\end{array}\right\}$. Then $A: H \rightarrow H$ and $A_{k}, B: C \rightarrow H$ are $\zeta$-inverse strongly monotone with $\zeta=(1 / 2), \zeta_{k}$-inverse strongly monotone with $\zeta_{k}=(1 / 2)$ and $\xi$-inverse-strongly monotone with $\xi=(1 / 2)$, respectively, for $k=1,2$. Also, put $\mu=2, Q=0, V=(3 / 2) S$, and $F=(1 / 2) I$, where $I$ is the identity mapping of $\mathbf{R}^{2}$. Then $V$ is a $\bar{\gamma}$-strongly positive bounded linear operator with $\bar{\gamma}=(3 / 2)$. It is easy to see that $\Omega:=\operatorname{GMEP}(\Theta, \varphi, A) \cap \operatorname{SGEP}(G) \cap I(B, R) \cap \Gamma=\{0\}$ where $G$ is defined as in Proposition CY. Let $\left\{\alpha_{n}\right\} \subset(0,1]$, $\left\{r_{n}\right\} \subset(0,2 \zeta]$, and $\left\{\rho_{n}\right\} \subset(0,2 \xi]$ with $\zeta=\xi=(1 / 2)$; that is, $\left\{\alpha_{n}\right\},\left\{\rho_{n}\right\},\left\{r_{n}\right\} \subset(0,1]$. In this case, for any given $x_{1} \in C$, the iterative scheme (119) is equivalent to the following one:

$$
\begin{aligned}
u_{n}= & S_{r_{n}}^{(\Theta, \varphi)}\left(I-r_{n} A\right) x_{n}=P_{C}\left(I-r_{n} A\right) x_{n}=\left(1-r_{n}\right) x_{n}, \\
v_{n}= & J_{R, \rho_{n}}\left(I-\rho_{n} B\right) u_{n}=J_{R, \rho_{n}} u_{n} \\
= & P_{C}\left(u_{n}-\rho_{n} S v_{n}\right)=u_{n}-\rho_{n} v_{n}, \\
x_{n}= & \left(I-s_{n} V\right) T_{n} G v_{n} \\
& +s_{n}\left[T_{n} x_{n}-\alpha_{n}\left(\mu F\left(T_{n} x_{n}\right)-\gamma Q x_{n}\right)\right] \\
= & \left(I-\frac{3}{2} s_{n} S\right) T_{n} P_{C}\left(I-v_{1} A_{1}\right) P_{C}\left(I-v_{2} A_{2}\right) v_{n} \\
& +s_{n}\left[T_{n} x_{n}-\alpha_{n} \mu F\left(T_{n} x_{n}\right)\right] \\
= & \left(I-\frac{3}{2} s_{n} S\right) T_{n} v_{n}+s_{n}\left[T_{n} x_{n}-\alpha_{n} T_{n} x_{n}\right] \\
= & \left(I-\frac{3}{2} s_{n} S\right) T_{n} v_{n}+s_{n}\left(1-\alpha_{n}\right) T_{n} x_{n}, \quad \forall n \geq 1,
\end{aligned}
$$

where $P_{C}\left(I-\lambda_{n} \nabla f\right)=s_{n} I+\left(1-s_{n}\right) T_{n}$ and $s_{n}=\left(\left(2-\lambda_{n} L\right) / 4\right) \epsilon$ $(0,(1 / 2))$ for each $\lambda_{n} \in(0,(2 / L))$ with $L=4$; that is, $s_{n}=$ $\left(\left(1-2 \lambda_{n}\right) / 2\right) \in(0,(1 / 2))$ for each $\lambda_{n} \in(0,(1 / 2))$.

Next, taking into account $v_{n}=J_{R, \rho_{n}} u_{n}=\left(I+\rho_{n} R\right)^{-1} u_{n}$, we get $u_{n} \in v_{n}+\rho_{n} R v_{n}$, which leads to

$$
\frac{u_{n}-v_{n}}{\rho_{n}} \in R v_{n}=S v_{n}+N_{C} v_{n}
$$

Hence, we have $\left\langle v_{n}-u,\left(\left(u_{n}-v_{n}\right) / \rho_{n}\right)-S v_{n}\right\rangle \geq 0$, for all $u \in C$; that is,

$$
\left\langle u-v_{n}, u_{n}-\rho_{n} S v_{n}-v_{n}\right\rangle \leq 0, \quad \forall u \in C,
$$

which immediately implies that $v_{n}=P_{C}\left(u_{n}-\rho_{n} S v_{n}\right)=u_{n}-$ $\rho_{n} v_{n}$. Thus,

$$
v_{n}=\frac{1}{1+\rho_{n}} u_{n}=\frac{1-r_{n}}{1+\rho_{n}} x_{n} .
$$

Also, note that

$$
\left(1-\lambda_{n}\right) x_{n}=P_{C}\left(I-\lambda_{n} \nabla f\right) x_{n}=s_{n} x_{n}+\left(1-s_{n}\right) T_{n} x_{n} .
$$


So, we obtain

$$
T_{n} x_{n}=\frac{1-s_{n}-\lambda_{n}}{1-s_{n}} x_{n}
$$

On the other hand, we have

$$
\left(1-\lambda_{n}\right) v_{n}=P_{C}\left(I-\lambda_{n} \nabla f\right) v_{n}=s_{n} v_{n}+\left(1-s_{n}\right) T_{n} v_{n}
$$

and hence

$$
T_{n} v_{n}=\frac{1-s_{n}-\lambda_{n}}{1-s_{n}} v_{n}=\frac{1-s_{n}-\lambda_{n}}{1-s_{n}} \cdot \frac{1-r_{n}}{1+\rho_{n}} x_{n},
$$

which together with (124) implies that

$$
\begin{aligned}
x_{n}= & \left(I-\frac{3}{2} s_{n} S\right) T_{n} v_{n}+s_{n}\left(1-\alpha_{n}\right) T_{n} x_{n} \\
= & \left(I-\frac{3}{2} s_{n} S\right) \frac{1-s_{n}-\lambda_{n}}{1-s_{n}} \frac{1-r_{n}}{1+\rho_{n}} x_{n} \\
& +s_{n}\left(1-\alpha_{n}\right) \frac{1-s_{n}-\lambda_{n}}{1-s_{n}} x_{n} \\
= & \left(1-\frac{3}{2} s_{n}\right) \frac{1-s_{n}-\lambda_{n}}{1-s_{n}} \frac{1-r_{n}}{1+\rho_{n}} x_{n} \\
& +s_{n}\left(1-\alpha_{n}\right) \frac{1-s_{n}-\lambda_{n}}{1-s_{n}} x_{n} \\
= & {\left[\left(1-\frac{3}{2} s_{n}\right) \frac{1-r_{n}}{1+\rho_{n}}+s_{n}\left(1-\alpha_{n}\right)\right] \frac{1-s_{n}-\lambda_{n}}{1-s_{n}} x_{n} . }
\end{aligned}
$$

It is clear that if $s_{n}=\left(\left(1-2 \lambda_{n}\right) / 2\right)=\left(\left(1-2 \lambda_{n}\right) / 2\right) \in(0,(1 / 2))$ for each $\lambda_{n} \in(0,(1 / 2))$, then, for $\left\{r_{n}\right\},\left\{\alpha_{n}\right\},\left\{\rho_{n}\right\} \subset(0,1]$, we deduce from (132) that

$$
\begin{aligned}
\left\|x_{n}\right\| & =\left[\left(1-\frac{3}{2} s_{n}\right) \frac{1-r_{n}}{1+\rho_{n}}+s_{n}\left(1-\alpha_{n}\right)\right] \frac{1-s_{n}-\lambda_{n}}{1-s_{n}}\left\|x_{n}\right\| \\
& \leq\left(1-\frac{3}{2} s_{n}\right) \frac{1-r_{n}}{1+\rho_{n}}+s_{n}\left(1-\alpha_{n}\right)\left\|x_{n}\right\| \\
& \leq\left(1-\frac{3}{2} s_{n}+s_{n}\right)\left\|x_{n}\right\| \\
& =\left(1-\frac{1}{2} s_{n}\right)\left\|x_{n}\right\| .
\end{aligned}
$$

Therefore, $x_{n}=0$ for all $n \geq 1$. There is no doubt that $\left\{x_{n}\right\}$ converges to the unique element 0 in $\Omega$, which solves the VIP (121).

\section{Fixed Point Problems with Constraints}

In this section, we will introduce and analyze another implicit iterative algorithm for solving the fixed point problem of infinitely many nonexpansive mappings with constraints of several problems: the GMEP (4), the SGEP (8), and finitely many variational inclusions in a real Hilbert space. We prove strong convergence theorem for the iterative algorithm under mild assumptions.

Theorem 23. Let $C$ be a nonempty closed convex subset of a real Hilbert space $H$. Let $N$ be an integer. Let $\Theta, \Theta_{1}, \Theta_{2}$ be three bifunctions from $\mathrm{C} \times \mathrm{C}$ to $\mathbf{R}$ satisfying $(\mathrm{H1})-(\mathrm{H} 4)$ and $\varphi: C \rightarrow$ $\mathbf{R}$ a lower semicontinuous and convex functional. Let $R_{i}: C \rightarrow$ $2^{H}$ be a maximal monotone mapping and let $A: H \rightarrow H$ and $A_{k}, B_{i}: C \rightarrow H$ be $\zeta$-inverse strongly monotone, $\zeta_{k^{-}}$ inverse strongly monotone, and $\eta_{i}$-inverse-strongly monotone, respectively, for $k=1,2$ and $i=1,2, \ldots, N$. Let $\left\{T_{n}\right\}_{n=1}^{\infty}$ be a sequence of nonexpansive mappings on $H$ and $\left\{\lambda_{n}\right\}$ a sequence in $(0, b]$ for some $b \in(0,1)$. Let $V$ be a $\bar{\gamma}$-strongly positive bounded linear operator with $\bar{\gamma}>1$. Let $F: H \rightarrow H$ be a $\kappa$-Lipschitzian and $\eta$-strongly monotone operator with positive constants $\kappa, \eta>0$. Let $Q: H \rightarrow H$ be an l-Lipschitzian mapping with constant $l \geq 0$. Let $0<\mu<\left(2 \eta / \kappa^{2}\right)$ and $0 \leq \gamma l<\tau$, where $\tau=1-\sqrt{1-\mu\left(2 \eta-\mu \kappa^{2}\right)}$. Assume that $\Omega:=$ $\cap_{n=1}^{\infty} \operatorname{Fix}\left(T_{n}\right) \cap \operatorname{GMEP}(\Theta, \varphi, A) \cap \operatorname{SGEP}(G) \cap \cap_{i=1}^{N} I\left(B_{i}, R_{i}\right) \neq \emptyset$ where $G$ is defined as in Proposition CY. Let $\left\{r_{n}\right\}$ be a sequence in $(0,2 \zeta]$ and let $\left\{\alpha_{n}\right\}$ and $\left\{\sigma_{n}\right\}$ be sequences in $(0,1]$. Let $\left\{x_{n}\right\}$ be the sequence generated by

$$
\begin{aligned}
u_{n}= & S_{r_{n}}^{(\Theta, \varphi)}\left(I-r_{n} A\right) x_{n}, \\
v_{n}= & J_{R_{N}, \lambda_{N, n}}\left(I-\lambda_{N, n} B_{N}\right) J_{R_{N-1}, \lambda_{N-1, n}} \\
& \times\left(I-\lambda_{N-1, n} B_{N-1}\right) \cdots J_{R_{1}, \lambda_{1, n}}\left(I-\lambda_{1, n} B_{1}\right) u_{n}, \\
x_{n}= & \left(I-\sigma_{n} V\right) W_{n} G v_{n} \\
& +\sigma_{n}\left[W_{n} x_{n}-\alpha_{n}\left(\mu F\left(W_{n} x_{n}\right)-\gamma Q x_{n}\right)\right], \quad \forall n \geq 1,
\end{aligned}
$$

where $W_{n}$ is the $W$-mapping defined by (9). Suppose that the following conditions hold:

(i) $K: H \rightarrow \mathbf{R}$ is strongly convex with constant $\sigma>0$ and its derivative $K^{\prime}$ is Lipschitz continuous with constant $v>0$ such that the function $x \mapsto\left\langle y-x, K^{\prime}(x)\right\rangle$ is weakly upper semicontinuous for each $y \in H$;

(ii) for each $x \in H$, there exist a bounded subset $D_{x} \subset C$ and $z_{x} \in C$ such that for any $y \notin D_{x}$,

$\Theta\left(y, z_{x}\right)+\varphi\left(z_{x}\right)-\varphi(y)+\frac{1}{r}\left\langle K^{\prime}(y)-K^{\prime}(x), z_{x}-y\right\rangle<0 ;$

(iii) $\lim _{n \rightarrow \infty} \alpha_{n}=0$ and $\lim _{n \rightarrow \infty} \sigma_{n}=0$;

(iv) $v_{k} \in\left(0,2 \zeta_{k}\right), k=1,2$ and $\left\{\lambda_{i, n}\right\} \subset\left[a_{i}, b_{i}\right] \subset$ $\left(0,2 \eta_{i}\right), i=1,2, \ldots, N$;

(v) $0<\liminf _{n \rightarrow \infty} r_{n} \leq \lim \sup _{n \rightarrow \infty} r_{n}<2 \zeta$.

Assume that $S_{r}^{(\Theta, \varphi)}$ is firmly nonexpansive. Then $\left\{x_{n}\right\}$ converges strongly to a point $q \in \Omega$, which is a unique solution in $\Omega$ to the VIP:

$$
\langle(I-V) q, p-q\rangle \leq 0, \quad \forall p \in \Omega .
$$


Proof. First of all, let us show that the sequence $\left\{x_{n}\right\}$ is well defined. As $\lim _{n \rightarrow \infty} \sigma_{n}=0$ and $0<\liminf _{n \rightarrow \infty} r_{n} \leq$ $\limsup _{n \rightarrow \infty} r_{n}<2 \zeta$, we may assume, without loss of generality, that $\left\{r_{n}\right\} \subset[a, \widehat{a}] \subset(0,2 \zeta)$ and $\sigma_{n}\|V\| \leq 1$ for all $n \geq 1$. Utilizing the arguments similar to those in the proof of Theorem 19, we get

$$
\left\|I-\sigma_{n} V\right\| \leq 1-\sigma_{n} \bar{\gamma}
$$

Put

$$
\begin{aligned}
\Lambda_{n}^{i}= & J_{R_{i}, \lambda_{i, n}}\left(I-\lambda_{i, n} B_{i}\right) J_{R_{i-1}, \lambda_{i-1, n}} \\
& \times\left(I-\lambda_{i-1, n} B_{i-1}\right) \cdots J_{R_{1}, \lambda_{1, n}}\left(I-\lambda_{1, n} B_{1}\right)
\end{aligned}
$$

for all $i \in\{1,2, \ldots, N\}$, and $\Lambda_{n}^{0}=I$, where $I$ is the identity mapping on $H$. Then we have $v_{n}=\Lambda_{n}^{N} u_{n}$.

Consider the following mapping $G_{n}$ on $H$ defined by

$$
\begin{aligned}
G_{n} x= & \left(I-\sigma_{n} V\right) T_{n} G \Lambda_{n}^{N} S_{r_{n}}^{(\Theta, \varphi)}\left(I-r_{n} A\right) x \\
& +\sigma_{n}\left[T_{n} x-\alpha_{n}\left(\mu F\left(T_{n} x\right)-\gamma Q x\right)\right], \quad \forall x \in H, n \geq 1 .
\end{aligned}
$$

Since $A_{k}$ is $\zeta_{k}$-inverse-strongly monotone with $0 \leq \nu_{k} \leq 2 \zeta_{k}$ for $k=1,2$, repeating the same arguments as in the proof of Theorem 19 we deduce that $G$ is a nonexpansive mapping on $H$. Utilizing the arguments similar to those in the proof of Theorem 19, we deduce that, for any $x, y \in H$,

$$
\left\|G_{n} x-G_{n} y\right\|^{2} \leq\left(1-\sigma_{n} \alpha_{n}(\tau-\gamma l)\right)\|x-y\| .
$$

Since $0<1-\sigma_{n} \alpha_{n}(\tau-\gamma l)<1, G_{n}: H \rightarrow H$ is a contraction. Therefore, by the Banach contraction principle, $G_{n}$ has a unique fixed point $x_{n} \in H$, which uniquely solves the fixed point equation

$$
\begin{aligned}
x_{n}= & \left(I-\sigma_{n} V\right) W_{n} G v_{n} \\
& +\sigma_{n}\left[W_{n} x_{n}-\alpha_{n}\left(\mu F\left(W_{n} x_{n}\right)-\gamma Q x_{n}\right)\right] .
\end{aligned}
$$

This shows that the sequence $\left\{x_{n}\right\}$ is defined well.

It is easy to see the VIP (43) has only one solution in $\Omega$. Below we use $q \in \Omega$ to denote the unique solution of the VIP (43).

Now, let us show that $\left\{x_{n}\right\}$ is bounded. In fact, take $p \in \Omega$ arbitrarily. Repeating the same arguments as in the proof of Theorem 19 we obtain

$$
\begin{aligned}
\left\|u_{n}-p\right\|^{2} & \leq\left\|x_{n}-p\right\|^{2}+r_{n}\left(r_{n}-2 \zeta\right)\left\|A x_{n}-A p\right\|^{2}, \\
\left\|v_{n}-p\right\|^{2} & \leq\left\|\Lambda_{n}^{i} u_{n}-p\right\|^{2} \\
& \leq\left\|u_{n}-p\right\|^{2}+\lambda_{i, n}\left(\lambda_{i, n}-2 \eta_{i}\right)\left\|B_{i} \Lambda_{n}^{i-1} u_{n}-B_{i} p\right\|^{2},
\end{aligned}
$$

$$
\begin{aligned}
& \left\|G v_{n}-p\right\|^{2} \\
& \leq\left\|T_{v_{2}}^{\Theta_{2}}\left(I-v_{2} A_{2}\right) v_{n}-T_{v_{2}}^{\Theta_{2}}\left(I-v_{2} A_{2}\right) p\right\|^{2} \\
& +v_{1}\left(v_{1}-2 \zeta_{1}\right) \\
& \times\left\|A_{1} T_{v_{2}}^{\Theta_{2}}\left(I-v_{2} A_{2}\right) v_{n}-A_{1} T_{v_{2}}^{\Theta_{2}}\left(I-v_{2} A_{2}\right) p\right\|^{2} \\
& \leq\left\|v_{n}-p\right\|^{2}+v_{2}\left(v_{2}-2 \zeta_{2}\right)\left\|A_{2} v_{n}-A_{2} p\right\|^{2}, \\
& \left\|x_{n}-p\right\|^{2} \leq\left(1-\sigma_{n} \bar{\gamma}\right)\left\|G v_{n}-p\right\|^{2}+\sigma_{n}\left\|x_{n}-p\right\|^{2} \\
& +2 s_{n}\left\langle(I-V) p+\alpha_{n}(\gamma Q-\mu F) p, x_{n}-p\right\rangle \\
& \leq\left(1-\sigma_{n} \bar{\gamma}\right)\left\|v_{n}-p\right\|^{2}+\sigma_{n}\left\|x_{n}-p\right\|^{2} \\
& +2 s_{n}\left\langle(I-V) p+\alpha_{n}(\gamma Q-\mu F) p, x_{n}-p\right\rangle \\
& =\left(1-\sigma_{n}(\bar{\gamma}-1)\right)\left\|x_{n}-p\right\|^{2} \\
& +2 \sigma_{n}(\|(I-V) p\|+\|(\gamma Q-\mu F) p\|) \\
& \times\left\|x_{n}-p\right\| \text {. }
\end{aligned}
$$

From (145) we conclude that

$$
\left\|x_{n}-p\right\| \leq \frac{2}{\bar{\gamma}-1}(\|(I-V) p\|+\|(\gamma Q-\mu F) p\|) .
$$

Hence $\left\{x_{n}\right\}$ is bounded. So, according to (142) and (143) we know that $\left\{u_{n}\right\},\left\{v_{n}\right\},\left\{G v_{n}\right\},\left\{W_{n} G v_{n}\right\},\left\{W_{n} x_{n}\right\},\left\{Q x_{n}\right\}$, and $\left\{F W_{n} x_{n}\right\}$ are bounded.

Repeating the arguments similar to those of (68), (76), (78), (93), and (97) in the proof of Theorem 19 we obtain that $\left\|x_{n}-u_{n}\right\| \rightarrow 0,\left\|\Lambda_{n}^{i-1} u_{n}-\Lambda_{n}^{i} u_{n}\right\| \rightarrow 0,\left\|x_{n}-v_{n}\right\| \rightarrow 0$, $\left\|v_{n}-G v_{n}\right\| \rightarrow 0$ and $\left\|v_{n}-W_{n} v_{n}\right\| \rightarrow 0$ as $n \rightarrow \infty$. In addition, note that

$$
\left\|v_{n}-W v_{n}\right\| \leq\left\|v_{n}-W_{n} v_{n}\right\|+\left\|W_{n} v_{n}-W v_{n}\right\| .
$$

So, from $\left\|v_{n}-W_{n} v_{n}\right\| \rightarrow 0$ and [15, Remark 3.2] it follows that

$$
\lim _{n \rightarrow \infty}\left\|v_{n}-W v_{n}\right\|=0
$$

Further, we show that $\omega_{w}\left(x_{n}\right) \subset \Omega$. Indeed, since $\left\{x_{n}\right\}$ is bounded, there exists a subsequence $\left\{x_{n_{i}}\right\}$ of $\left\{x_{n}\right\}$ which converges weakly to some $w$. Note that $\lim _{n \rightarrow \infty}\left\|x_{n}-u_{n}\right\|=0$. Hence $u_{n_{i}} \rightarrow w$. Since $C$ is closed and convex, $C$ is weakly closed. So, we have $w \in C$. On the other hand, it is easy to see that $u_{n_{i}} \rightarrow w, v_{n_{i}} \rightarrow w$, and $\Lambda_{n_{i}}^{m} u_{n_{i}} \rightarrow w$, where $m \in\{1,2, \ldots, N\}$. Repeating the same arguments as in the proof of Theorem 19, we obtain that $w \in \cap_{i=1}^{N} I\left(B_{i}, R_{i}\right) \cap$ $\operatorname{GMEP}(\Theta, \varphi, A)$. Next let us show that $w \in \operatorname{SGEP}(G) \cap$ $\cap_{n=1}^{\infty} \operatorname{Fix}\left(T_{n}\right)$. As a matter of fact, from $\left\|v_{n}-G v_{n}\right\| \rightarrow 0$, $\left\|v_{n}-W v_{n}\right\| \rightarrow 0, v_{n_{i}} \rightarrow w$ and Lemma 10, we deduce that $w \in$ $\operatorname{SGEP}(G)$ and $w \in \operatorname{Fix}(W)=\cap_{n=1}^{\infty} \operatorname{Fix}\left(T_{n}\right)$ (due to Lemma 12). Hence we get $w \in \operatorname{SGEP}(G) \cap \cap_{n=1}^{\infty} \operatorname{Fix}\left(T_{n}\right)$. Therefore, $w \in$ $\cap_{n=1}^{\infty} \operatorname{Fix}\left(T_{n}\right) \cap \operatorname{GMEP}(\Theta, \varphi, A) \cap \operatorname{SGEP}(G) \cap \cap_{i=1}^{N} I\left(B_{i}, R_{i}\right)=: \Omega$. This shows that $\omega_{w}\left(x_{n}\right) \subset \Omega$. 
Finally, we prove that $\left\{x_{n}\right\}$ converges strongly to $q \in \Omega$, which is the unique solution in $\Omega$ to the VIP (136). In fact, we note that, for $w \in \Omega$ with $x_{n_{i}} \rightarrow w$,

$$
\begin{aligned}
x_{n}-w= & \left(I-\sigma_{n} V\right)\left(W_{n} G v_{n}-w\right) \\
& +\sigma_{n}\left[\alpha_{n} \gamma\left(Q x_{n}-Q w\right)\right. \\
& \left.\quad+\left(I-\alpha_{n} \mu F\right) W_{n} x_{n}-\left(I-\alpha_{n} \mu F\right) W_{n} w\right] \\
& +\sigma_{n}\left[(I-V) w+\alpha_{n}(\gamma Q-\mu F) w\right] .
\end{aligned}
$$

Utilizing the arguments similar to those in the proof of Theorem 19, we obtain that

$$
\begin{aligned}
\left\|x_{n}-w\right\|^{2} \leq & \left(1-\sigma_{n}(\bar{\gamma}-1)\right)\left\|x_{n}-w\right\|^{2} \\
& +\sigma_{n}\left\langle(I-V) w+\alpha_{n}(\gamma Q-\mu F) w, x_{n}-w\right\rangle,
\end{aligned}
$$

which hence leads to

$$
\left\|x_{n}-w\right\|^{2} \leq \frac{\left\langle(I-V) w+\alpha_{n}(\gamma Q-\mu F) w, x_{n}-w\right\rangle}{\bar{\gamma}-1} .
$$

In particular, we have

$$
\left\|x_{n_{i}}-w\right\|^{2} \leq \frac{\left\langle(I-V) w+\alpha_{n_{i}}(\gamma Q-\mu F) w, x_{n_{i}}-w\right\rangle}{\bar{\gamma}-1} .
$$

Since $x_{n_{i}} \rightarrow w$ and $\lim _{n \rightarrow \infty} \alpha_{n}=0$, it follows from (152) that $x_{n_{i}} \rightarrow w$ as $i \rightarrow \infty$.

Now we show that $w$ solves the VIP (136). As a matter of fact, from (142) and (145) we obtain that, for any $p \in \Omega$,

$$
\begin{aligned}
\left\|x_{n}-p\right\|^{2} \leq & \left(1-\sigma_{n} \bar{\gamma}\right)\left\|u_{n}-p\right\|^{2}+\sigma_{n}\left\|x_{n}-p\right\|^{2} \\
& +2 \sigma_{n}\left\langle(I-V) p+\alpha_{n}(\gamma Q-\mu F) p, x_{n}-p\right\rangle \\
\leq & \left(1-\sigma_{n} \bar{\gamma}\right)\left\|x_{n}-p\right\|^{2}+\sigma_{n}\left\|x_{n}-p\right\|^{2} \\
& +2 \sigma_{n}\left\langle(I-V) p+\alpha_{n}(\gamma Q-\mu F) p, x_{n}-p\right\rangle \\
= & \left(1-\sigma_{n}(\bar{\gamma}-1)\right)\left\|x_{n}-p\right\|^{2} \\
& +2 \sigma_{n}\left\langle(I-V) p+\alpha_{n}(\gamma Q-\mu F) p, x_{n}-p\right\rangle \\
\leq & \left\|x_{n}-p\right\|^{2} \\
& +2 \sigma_{n}\left\langle(I-V) p+\alpha_{n}(\gamma Q-\mu F) p, x_{n}-p\right\rangle,
\end{aligned}
$$

which immediately implies that

$$
\left\langle(V-I) p+\alpha_{n}(\mu F-\gamma Q) p, x_{n}-p\right\rangle \leq 0 \text {. }
$$

Since $\alpha_{n} \rightarrow 0$ and $x_{n_{i}} \rightarrow w$, we get

$$
\langle(V-I) p, w-p\rangle \leq 0, \quad \forall p \in \Omega \text {. }
$$

By Minty's lemma, $w$ is a solution in $\Omega$ to the VIP (136). In terms of the uniqueness of solutions of VIP (136), we deduce that $w=q$ and $x_{n_{i}} \rightarrow q$ as $n \rightarrow \infty$. So, every weak convergence subsequence of $\left\{x_{n}\right\}$ converges strongly to the unique solution $q$ of VIP (136). Therefore, $\left\{x_{n}\right\}$ converges strongly to the unique solution $q$ of VIP (136). This completes the proof.

Corollary 24. Let $C$ be a nonempty closed convex subset of a real Hilbert space $H$. Let $\Theta, \Theta_{1}, \Theta_{2}$ be three bifunctions from $\mathrm{C} \times \mathrm{C}$ to $\mathbf{R}$ satisfying (H1)-(H4) and let $\varphi: C \rightarrow \mathbf{R}$ be a lower semicontinuous and convex functional. Let $R_{i}: C \rightarrow 2^{H}$ be a maximal monotone mapping and let $A, A_{k}: H \rightarrow$ $H$ and $B_{i}: C \rightarrow H$ be $\zeta$-inverse strongly monotone, $\zeta_{k^{-}}$ inverse strongly monotone, and $\eta_{i}$-inverse strongly monotone, respectively, for $k=1,2$ and $i=1,2$. Let $\left\{T_{n}\right\}_{n=1}^{\infty}$ be a sequence of nonexpansive mappings on $H$ and let $\left\{\lambda_{n}\right\}$ be a sequence in $(0, b]$ for some $b \in(0,1)$. Let $V$ be a $\bar{\gamma}$-strongly positive bounded linear operator with $\bar{\gamma}>1$. Let $F: H \rightarrow H$ be a $\kappa$-Lipschitzian and $\eta$-strongly monotone operator with positive constants $\kappa, \eta>0$. Let $Q: H \rightarrow H$ be an l-Lipschitzian mapping with constant $l \geq 0$. Let $0<\mu<\left(2 \eta / \kappa^{2}\right)$ and $0 \leq \gamma l<\tau$, where $\tau=1-\sqrt{1-\mu\left(2 \eta-\mu \kappa^{2}\right)}$. Assume that $\Omega:=\cap_{n=1}^{\infty} \operatorname{Fix}\left(T_{n}\right) \cap \operatorname{GMEP}(\Theta, \varphi, A) \cap \operatorname{SGEP}(G) \cap I\left(B_{1}, R_{1}\right) \cap$ $I\left(B_{2}, R_{2}\right) \neq \emptyset$ where $G$ is defined as in Proposition CY. Let $\left\{r_{n}\right\}$ be a sequence in $(0,2 \zeta]$ and $\left\{\alpha_{n}\right\}$ and $\left\{\sigma_{n}\right\}$ sequences in $(0,1]$. Let $\left\{x_{n}\right\}$ be the sequence generated by

$$
\begin{aligned}
u_{n}= & S_{r_{n}}^{(\Theta, \varphi)}\left(I-r_{n} A\right) x_{n}, \\
v_{n}= & J_{R_{2}, \lambda_{2, n}}\left(I-\lambda_{2, n} B_{2}\right) J_{R_{1}, \lambda_{1, n}}\left(I-\lambda_{1, n} B_{1}\right) u_{n}, \\
x_{n}= & \left(I-\sigma_{n} V\right) W_{n} G v_{n} \\
& +\sigma_{n}\left[W_{n} x_{n}-\alpha_{n}\left(\mu F\left(W_{n} x_{n}\right)-\gamma Q x_{n}\right)\right], \quad \forall n \geq 1,
\end{aligned}
$$

where $W_{n}$ is the $W$-mapping defined by (9). Suppose that the following conditions hold:

(i) $\mathrm{K}: H \rightarrow \mathbf{R}$ is strongly convex with constant $\sigma>0$ and its derivative $K^{\prime}$ is Lipschitz continuous with constant $v>0$ such that the function $x \mapsto\left\langle y-x, K^{\prime}(x)\right\rangle$ is weakly upper semicontinuous for each $y \in H$;

(ii) for each $x \in H$, there exist a bounded subset $D_{x} \subset C$ and $z_{x} \in C$ such that, for any $y \notin D_{x}$,

$\Theta\left(y, z_{x}\right)+\varphi\left(z_{x}\right)-\varphi(y)+\frac{1}{r}\left\langle K^{\prime}(y)-K^{\prime}(x), z_{x}-y\right\rangle<0 ;$

(iii) $\lim _{n \rightarrow \infty} \alpha_{n}=0$ and $\lim _{n \rightarrow \infty} \sigma_{n}=0$;

(iv) $v_{k} \in\left(0,2 \zeta_{k}\right), k=1,2$ and $\left\{\lambda_{i, n}\right\} \subset\left[a_{i}, b_{i}\right] \subset$ $\left(0,2 \eta_{i}\right), i=1,2$;

(v) $0<\liminf _{n \rightarrow \infty} r_{n} \leq \lim \sup _{n \rightarrow \infty} r_{n}<2 \zeta$.

Assume that $S_{r}^{(\Theta, \varphi)}$ is firmly nonexpansive. Then $\left\{x_{n}\right\}$ converges strongly to a point $q \in \Omega$, which is a unique solution in $\Omega$ to the VIP:

$$
\langle(I-V) q, p-q\rangle \leq 0, \quad \forall p \in \Omega .
$$


Corollary 25. Let $C$ be a nonempty closed convex subset of a real Hilbert space $H$. Let $\Theta, \Theta_{1}, \Theta_{2}$ be three bifunctions from $\mathrm{C} \times \mathrm{C}$ to $\mathbf{R}$ satisfying (H1)-(H4) and $\varphi: C \rightarrow \mathbf{R}$ a lower semicontinuous and convex functional. Let $R: C \rightarrow 2^{H}$ be a maximal monotone mapping and let $A, A_{k}: H \rightarrow H$ and $B$ : $\mathrm{C} \rightarrow \mathrm{H}$ be $\zeta$-inverse strongly monotone, $\zeta_{k}$-inverse strongly monotone, and $\xi$-inverse strongly monotone, respectively, for $k=1,2$. Let $\left\{T_{n}\right\}_{n=1}^{\infty}$ be a sequence of nonexpansive mappings on $H$ and $\left\{\lambda_{n}\right\}$ a sequence in $(0, b]$ for some $b \in(0,1)$. Let $V$ be $a \bar{\gamma}$-strongly positive bounded linear operator with $\bar{\gamma}>1$. Let $F$ : $H \rightarrow H$ be a $\kappa$-Lipschitzian and $\eta$-strongly monotone operator with positive constants $\kappa, \eta>0$. Let $\mathrm{Q}: H \rightarrow H$ be an lLipschitzian mapping with constant $l \geq 0$. Let $0<\mu<\left(2 \eta / \kappa^{2}\right)$ and $0 \leq \gamma l<\tau$, where $\tau=1-\sqrt{1-\mu\left(2 \eta-\mu \kappa^{2}\right)}$. Assume that $\Omega:=\cap_{n=1}^{\infty} \operatorname{Fix}\left(T_{n}\right) \cap \operatorname{GMEP}(\Theta, \varphi, A) \cap \operatorname{SGEP}(G) \cap I(B, R) \neq \emptyset$ where $G$ is defined as in Proposition CY. Let $\left\{r_{n}\right\}$ be a sequence in $(0,2 \zeta]$ and $\left\{\alpha_{n}\right\}$ and $\left\{\sigma_{n}\right\}$ sequences in $(0,1]$. Let $\left\{x_{n}\right\}$ be the sequence generated by

$$
\begin{aligned}
u_{n}= & S_{r_{n}}^{(\Theta, \varphi)}\left(I-r_{n} A\right) x_{n}, \\
v_{n}= & J_{R, \rho_{n}}\left(I-\rho_{n} B\right) u_{n}, \\
x_{n}= & \left(I-\sigma_{n} V\right) W_{n} G v_{n} \\
& +\sigma_{n}\left[W_{n} x_{n}-\alpha_{n}\left(\mu F\left(W_{n} x_{n}\right)-\gamma Q x_{n}\right)\right], \quad \forall n \geq 1,
\end{aligned}
$$

where $W_{n}$ is the $W$-mapping defined by (9). Suppose that the following conditions hold:

(i) $K: H \rightarrow \mathbf{R}$ is strongly convex with constant $\sigma>0$ and its derivative $K^{\prime}$ is Lipschitz continuous with constant $v>0$ such that the function $x \mapsto\left\langle y-x, K^{\prime}(x)\right\rangle$ is weakly upper semicontinuous for each $y \in H$;

(ii) for each $x \in H$, there exist a bounded subset $D_{x} \subset C$ and $z_{x} \in C$ such that, for any $y \notin D_{x}$,

$\Theta\left(y, z_{x}\right)+\varphi\left(z_{x}\right)-\varphi(y)+\frac{1}{r}\left\langle K^{\prime}(y)-K^{\prime}(x), z_{x}-y\right\rangle<0 ;$

(iii) $\lim _{n \rightarrow \infty} \alpha_{n}=0$ and $\lim _{n \rightarrow \infty} \sigma_{n}=0$;

(iv) $\nu_{k} \in\left(0,2 \zeta_{k}\right), k=1,2$ and $\left\{\rho_{n}\right\} \subset[a, b] \subset(0,2 \xi)$;

(v) $0<\liminf _{n \rightarrow \infty} r_{n} \leq \limsup _{n \rightarrow \infty} r_{n}<2 \zeta$.

Assume that $S_{r}^{(\Theta, \varphi)}$ is firmly nonexpansive. Then $\left\{x_{n}\right\}$ converges strongly to a point $q \in \Omega$, which is a unique solution in $\Omega$ to the VIP:

$$
\langle(I-V) q, p-q\rangle \leq 0, \quad \forall p \in \Omega .
$$

\section{Conflict of Interests}

The authors declare that there is no conflict of interests regarding the publication of this paper.

\section{Acknowledgments}

This research was partially supported by the National Science Foundation of China (11071169), Innovation Program of Shanghai Municipal Education Commission (09ZZ133), and Ph.D. Program Foundation of Ministry of Education of China (20123127110002). This work was supported partly by the National Science Council of the Republic of China. This research was partially supported by a grant from NSC.

\section{References}

[1] J.-W. Peng and J.-C. Yao, "A new hybrid-extragradient method for generalized mixed equilibrium problems, fixed point problems and variational inequality problems," Taiwanese Journal of Mathematics, vol. 12, no. 6, pp. 1401-1432, 2008.

[2] L.-C. Ceng and J.-C. Yao, "A relaxed extragradient-like method for a generalized mixed equilibrium problem, a general system of generalized equilibria and a fixed point problem," Nonlinear Analysis: Theory, Methods \& Applications, vol. 72, no. 3-4, pp. 1922-1937, 2010.

[3] L.-C. Ceng, Q. H. Ansari, and S. Schaible, "Hybrid extragradient-like methods for generalized mixed equilibrium problems, systems of generalized equilibrium problems and optimization problems," Journal of Global Optimization, vol. 53, no. 1, pp. 69-96, 2012.

[4] N.-J. Huang, "A new completely general class of variational inclusions with noncompact valued mappings," Computers \& Mathematics with Applications, vol. 35, no. 10, pp. 9-14, 1998.

[5] L.-C. Ceng and S. Al-Homidan, "Algorithms of common solutions for generalized mixed equilibria, variational inclusions, and constrained convex minimization," Abstract and Applied Analysis, vol. 2014, Article ID 132053, 25 pages, 2014.

[6] I. Yamada, "The hybrid steepest-descent method for the variational inequality problems over the intersection of the fixedpoint sets of nonexpansive mappings," in Inherently Parallel Algorithms in Feasibility and Optimization and Their Applications, D. Batnariu, Y. Censor, and S. Reich, Eds., pp. 473-504, North-Holland, Amsterdam, The Netherlands, 2001.

[7] H.-K. Xu, "Averaged mappings and the gradient-projection algorithm," Journal of Optimization Theory and Applications, vol. 150, no. 2, pp. 360-378, 2011.

[8] C. Byrne, "A unified treatment of some iterative algorithms in signal processing and image reconstruction," Inverse Problems, vol. 20, no. 1, pp. 103-120, 2004.

[9] L.-C. Ceng and J.-C. Yao, "A hybrid iterative scheme for mixed equilibrium problems and fixed point problems," Journal of Computational and Applied Mathematics, vol. 214, no. 1, pp. 186201, 2008.

[10] K. Geobel and W. A. Kirk, Topics on Metric Fixed-Point Theory, Cambridge University Press, Cambridge, UK, 1990.

[11] J. G. O’Hara, P. Pillay, and H.-K. Xu, "Iterative approaches to convex feasibility problems in Banach spaces," Nonlinear Analysis: Theory, Methods \& Applications, vol. 64, no. 9, pp. 2022-2042, 2006.

[12] H. K. Xu and T. H. Kim, "Convergence of hybrid steepestdescent methods for variational inequalities," Journal of Optimization Theory and Applications, vol. 119, no. 1, pp. 185-201, 2003. 
[13] L.-C. Zeng, S.-M. Guu, and J.-C. Yao, "Characterization of $H$-monotone operators with applications to variational inclusions," Computers \& Mathematics with Applications, vol. 50, no. 3-4, pp. 329-337, 2005.

[14] L.-C. Ceng, Q. H. Ansari, M. M. Wong, and J.-C. Yao, "Mann type hybrid extragradient method for variational inequalities, variational inclusions and fixed point problems," Fixed Point Theory, vol. 13, no. 2, pp. 403-422, 2012.

[15] Y. Yao, Y.-C. Liou, and J.-C. Yao, "Convergence theorem for equilibrium problems and fixed point problems of infinite family of nonexpansive mappings," Fixed Point Theory and Applications, vol. 2007, Article ID 64363, 12 pages, 2007. 


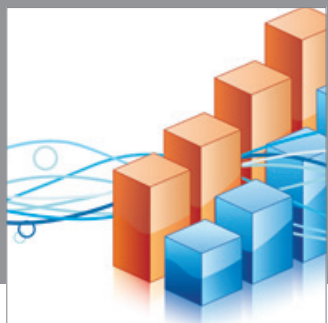

Advances in

Operations Research

mansans

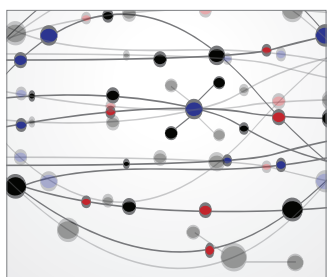

The Scientific World Journal
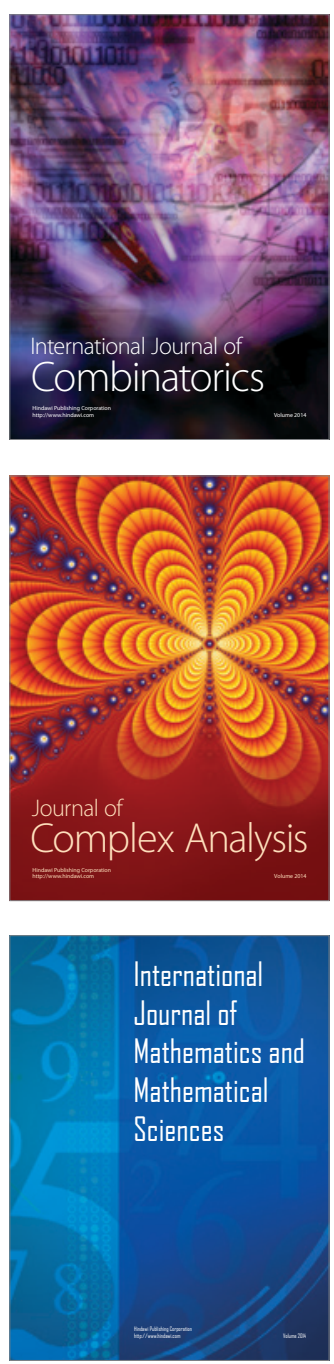
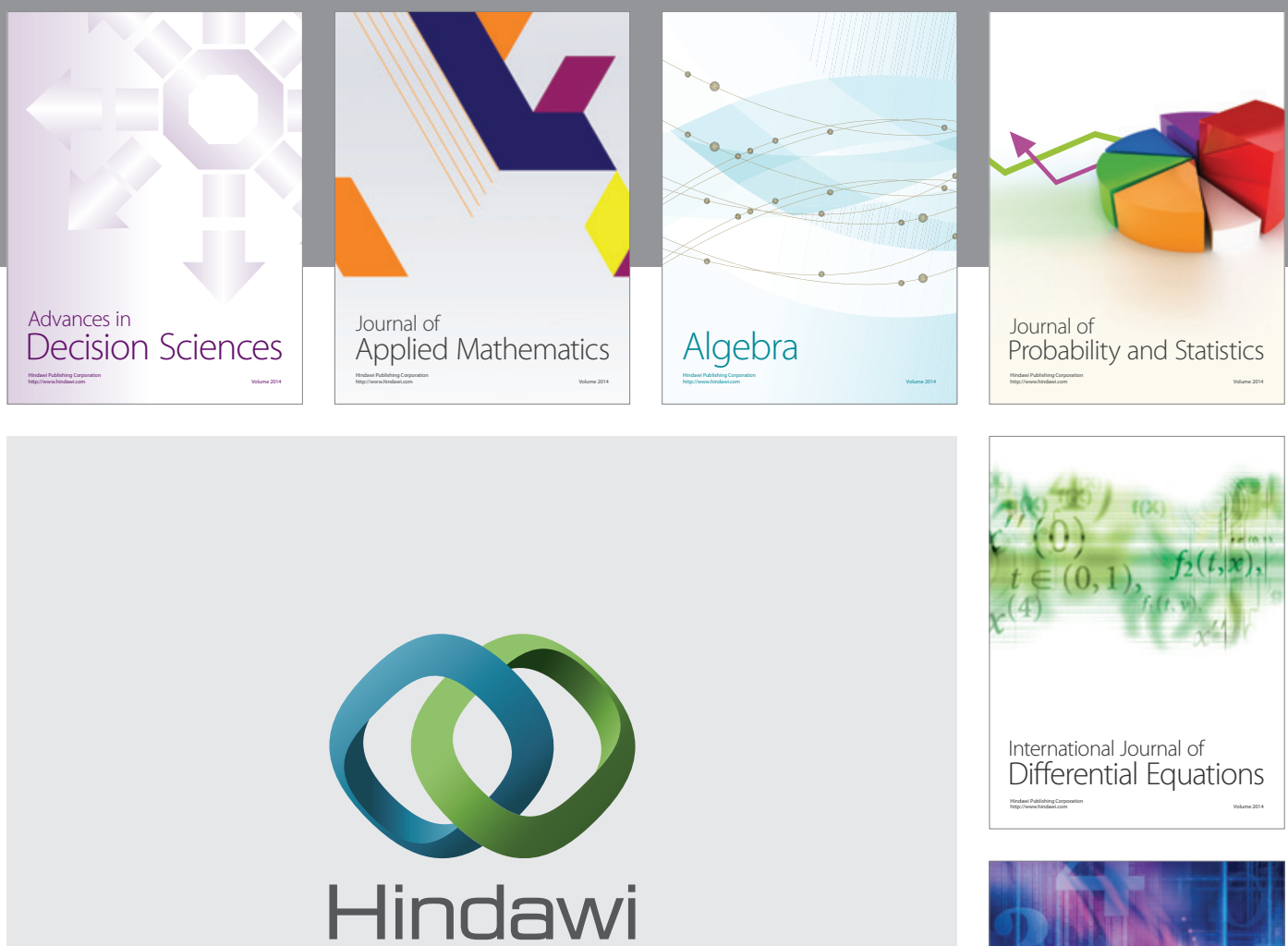

Submit your manuscripts at http://www.hindawi.com
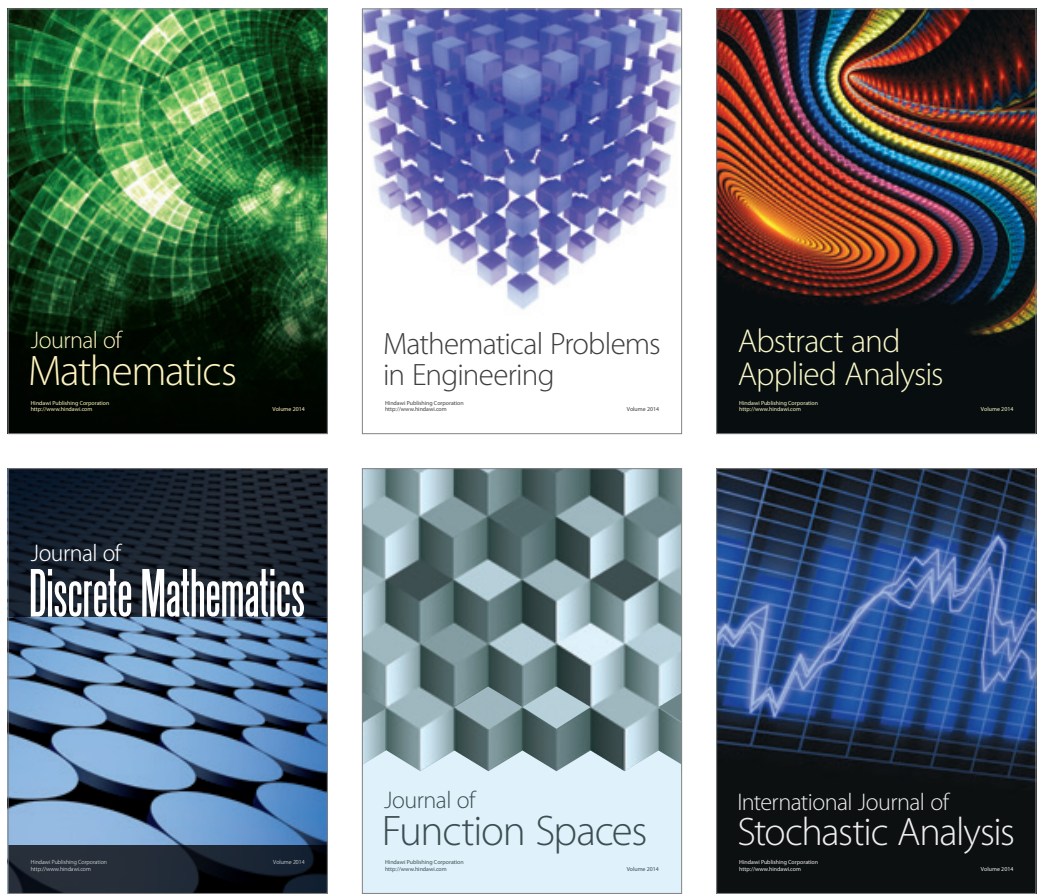

Journal of

Function Spaces

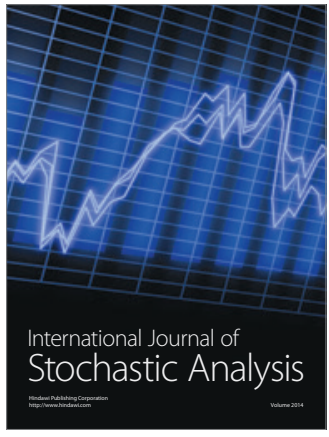

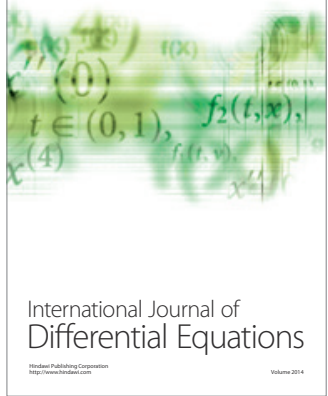
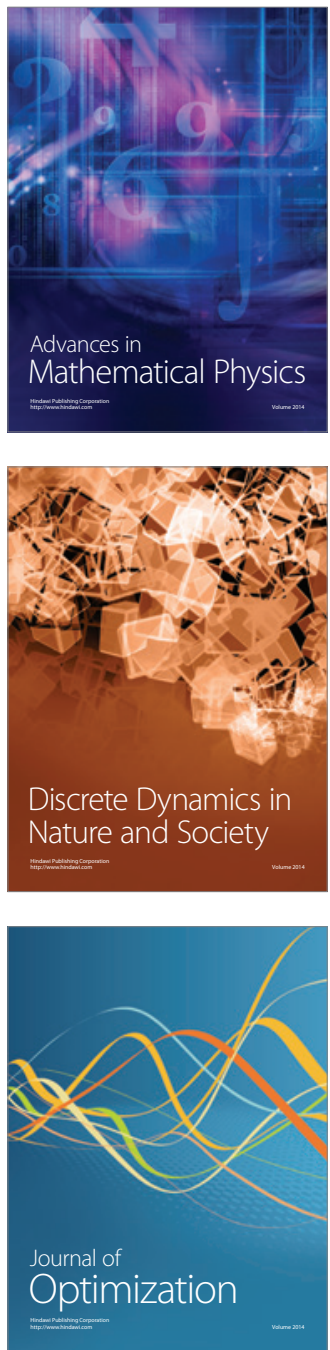